\title{
THE STRUCTURE OF $\omega_{1}$-SEPARABLE GROUPS ${ }^{1}$ \\ BY \\ PAUL C. EKLOF
}

\begin{abstract}
A classification theorem is proved for $\omega_{1}$-separable $\omega_{1}$-free abelian groups of cardinality $\omega_{1}$ assuming Martin's Axiom (MA) and $2^{\boldsymbol{\kappa}_{0}}>\boldsymbol{\kappa}_{1}$. As a consequence, several structural results about direct sum decompositions of $\omega_{1}$-separable groups are proved. These results are proved independent of ZFC, and, in addition, another structural property is proved undecidable in ZFC $+\mathrm{MA}+2^{\boldsymbol{N}_{0}}>$ $\boldsymbol{\kappa}_{1}$. The problem of classifying these groups in a model of $2^{\boldsymbol{N}_{0}}=\boldsymbol{N}_{1}$ is also investigated.
\end{abstract}

Introduction. Throughout this paper we shall use the term " $\omega_{1}$-separable group" to mean an abelian group such that every countable subset is contained in a countable free direct summand. In particular, such a group is $\omega_{1}$-free, i.e., every countable subgroup is free. (This is a more restricted usage than that in Fuchs [F, p. 121] but agrees with that in Griffith [G2, p. 102].) Obviously, $\omega_{1}$-separable groups are separable and homogeneous, so the results which follow provide a partial solution to Problem 77 of Fuchs [F, p. 184].

Griffith was the first to construct an $\omega_{1}$-separable group (of cardinality $\omega_{1}$ ) which is not free $[\mathbf{G 1}]$. Since then an unholy number of nonisomorphic $\omega_{1}$-separable groups of cardinality $\omega_{1}$ have been constructed (see [M1]). These are usually constructed by defining, by transfinite induction, an $\omega_{1}$-filtration of the group, i.e., a continuous chain $\left\{A_{\nu}: \nu<\omega\right\}$ of countable subgroups whose union is the group, $A$, such that each $A_{\nu+1}$ is a summand of $A$. If the set of $\nu$ such that $A / A_{\nu}$ is not $\omega_{1}$-free, is large enough (i.e., stationary in $\omega_{1}$ ) then $A$ is not free (see $\$ 1$ ). By this means one can, for example, construct a family of $2^{\boldsymbol{N}_{1}}$ nonisomorphic $\omega_{1}$-separable groups of cardinality $\omega_{1}$ which are pairwise quotient-equivalent, i.e., any two, $A$ and $B$, have $\omega_{1}$-filtrations $\left\{A_{\nu}: \nu<\omega_{1}\right\}$ and $\left\{B_{\nu}: \nu<\omega_{1}\right\}$ such that for all $\nu<\mu<\omega_{1}, A_{\mu} / A_{\nu} \cong B_{\mu} / B_{\nu}$ (cf. [E, Chapter 11] and Lemma 3.1 of this paper; see also [EMS, Theorem 3.3]).

In this paper we attempt to put some order into this apparent chaos by proving a classification theorem for $\omega_{1}$-separable groups of cardinality $\omega_{1}$ under the assumption of Martin's Axiom (MA) and the denial of the Continuum Hypothesis $(\neg \mathrm{CH})$ (see Theorem 1.2). We also show that this theorem fails in models of $\mathrm{CH}$ (Theorem 3.2) and, in fact, gives strong evidence that no useful classification of all $\omega_{1}$-separable groups of cardinality $\omega_{1}$ is possible in models of $\mathrm{CH}$ (Remark 3.3(1)). On the other hand, we show (Theorem 3.4) that there are models of $\mathrm{GCH}$ in which the

Received by the editors May 18, 1982.

1980 Mathematics Subject Classification. Primary 03E35, 20K20.

'Research partially supported by NSF Grant no. MCS-8003591.

(C) 1983 American Mathematical Society $0002-9947 / 83 \$ 1.00+\$ .25$ per page 
classification theorem holds in part, viz., it holds for a nontrivial initial segment of values of $\Gamma(A)$ (where $\Gamma(A)$ is a certain invariant of $A$-an equivalence class of subsets of $\omega_{1}($ see $\left.\S 1)\right)$.

The classification theorem says that (assuming MA $+\neg \mathrm{CH}$ ) two $\omega_{1}$-separable groups $A$ and $B$ are isomorphic iff they have $\omega_{1}$-filtrations $\left\{A_{\nu}: \nu<\omega_{1}\right\}$ and $\left\{B_{\nu}\right.$ : $\left.\nu<\omega_{1}\right\}$ all of whose proper initial segments are isomorphic, i.e., for all $\nu<\omega_{1}$ there is an isomorphism $f_{\nu}: A_{\nu} \rightarrow B_{\nu}$ such that for all $\mu<\nu, f_{\nu}\left(A_{\mu}\right)=B_{\mu}$. Thus the invariant of an $\omega_{1}$-separable group which classifies it is an equivalence class of $\omega_{1}$-filtrations (under the equivalence relation of isomorphism of all proper initial segments). In certain special cases this invariant can be described in a more concrete fashion (Theorem 1.4), but even in its general form the classification is a useful one. This is demonstrated by its application in $\$ 2$ to prove the following structural results (which are theorems of $\mathrm{ZFC}+\mathrm{MA}+\neg \mathrm{CH}$ but not of $\mathrm{ZFC}$ ).

(I) If $B \subseteq A$ are $\omega_{1}$-separable groups of cardinality $\omega_{1}$ and $A / B$ is the direct sum of a countable group and a free group, then $B \cong A$ (Corollary 2.5 ).

(II) If $A$ is $\omega_{1}$-separable of cardinality $\omega_{1}$, then $A \cong A \oplus \mathbf{Z}^{\left(\omega_{1}\right)}$ (Corollary 2.6).

(III) If $A$ is a nonfree $\omega_{1}$-separable group of cardinality $\omega_{1}$, then $A$ is the direct sum of $\omega_{1}$ nonfree subgroups (Theorem 2.8).

The above results are false in models of $V=L(3.7,3.6$ and 3.5 , respectively). It is open whether or not (II) and (III) are consistent with $\mathrm{ZFC}+\mathrm{CH}$ (but partial versions-for some values of $\Gamma(A)$-are consistent with $\mathrm{CH}$ (see Theorem 3.8)).

We also make use of the classification theorem to prove that certain questions about $\omega_{1}$-separable groups are undecidable even in $\mathrm{ZFC}+\mathrm{MA}+\neg \mathrm{CH}$. In particular, we consider a strengthening of property (III) above, in which we require that $A$ have direct decompositions corresponding to all possible partitions of $\Gamma(A)$ (see Definition 2.9). We show (Theorem 2.10) that the assertion that all $\omega_{1}$-separable groups of cardinality $\omega_{1}$ have this decomposition property is true in some models of $\mathrm{ZFC}+\mathrm{MA}+\neg \mathrm{CH}$ (constructed by proper forcing) and is false in others (constructed as c.c.c. extensions of $L$ ).

We shall make use of the following notational conventions: $A^{(\kappa)}$ denotes the direct sum of $\kappa$ copies of $A ;|A|$ denotes the cardinality of $A$; ZFC denotes the ZermeloFrankel axioms of set theory with the Axiom of Choice; $\mathrm{CH}$ is the Continuum Hypothesis; $\mathrm{GCH}$ is the Generalized Continuum Hypothesis; $V=L$ is the Axiom of Constructibility; $\omega_{1}$ and $\boldsymbol{N}_{1}$ are used, interchangeably, to denote the first uncountable cardinal. We use $Z=X \amalg Y$ to mean $Z=X \cup Y$ and $X \cap Y=\varnothing$. If $A$ is a torsion-free group and $X$ is a subset of $A,\langle X\rangle$ denotes the subgroup of $A$ generated by $X$, and $\langle X\rangle_{*}$ denotes the pure closure in $A$ of $\langle X\rangle$, i.e., $\langle X\rangle_{*}=\{a \in A \mid$ na $\in$ $\langle X\rangle$ for some $n \neq 0\}$. If $d \in \mathbf{Z}$ and $a \in A$, write $d \mid a$ in $A$ to mean $\exists x \in A$ s.t. $d x=a$.

I would like to express my thanks to M. Magidor, A. Mekler and S. Shelah for their invaluable help in the course of this work. I owe a special debt of gratitude to Wilfrid Hodges for his hospitality and assistance during the time I pursued this work while visiting Bedford College on sabbatical leave, 1980-81. In particular, I was aided greatly by the seminar in iterated and proper forcing held at Bedford College, and especially by the lucid seminar notes written by Wilfrid. 
Added in revision. A. Mekler has recently proved many of the results of this paper, under the assumption of PFA, for mixed $\omega_{1}$-separable groups, i.e., groups such that every countable subset is contained in a $\Sigma$-cyclic summand. In particular, he has proved a structure theorem for such groups from which such results as 2.6 and 2.10(2) (or their analogs) follow more easily. (For more details, see the Proceedings of the Honolulu Conference on Abelian Groups, December 28, 1982-January 4, 1983, Springer-Verlag Lecture Notes in Mathematics.)

0. Set-theoretic preliminaries. It is suggested that the reader read through Lemma 0.4 and then use the remainder of the section as reference, returning to it as needed for notions and results used in the rest of the paper. The reader is also referred to [ $\mathbf{J}$ or $\mathbf{E}$ ] for more detail about some of the definitions and theorems which follow.

Most of the sets we consider will be subsets of $\omega_{1}$, so we shall generally drop mention of $\omega_{1}$ and say e.g., "stationary" instead of "stationary in $\omega_{1}$."

A subset $C$ of $\omega_{1}$ is called a cub if it is unbounded in $\omega_{1}$ and closed in the order topology of $\omega_{1}$. For example, $\operatorname{Lim}\left(\omega_{1}\right)=\left\{\sigma<\omega_{1}: \sigma\right.$ is a limit ordinal $\}$ is a cub. A set $E \subseteq \omega_{1}$ is stationary if it has nonempty intersection with every cub; in particular, every cub is stationary (cf. [E, Lemma 1.1]). $E$ is costationary if $\omega_{1}-E$ is stationary. A fundamental fact about stationary sets (explaining their name) is the following [ $\mathbf{J}$, Theorem 22, p. 59]: A function $\theta: E \rightarrow \omega_{1}$ s.t. $\theta(\nu)<\nu$ for $\nu \in E$ is called regressive.

0.1 . Fodor's THEOREM. If $E$ is stationary and $\theta: E \rightarrow \omega_{1}$ such that $\theta$ is regressive, then there is a stationary $E_{0} \subseteq E$ and a $\gamma<\omega_{1}$ such that $\theta(\nu)=\gamma$ for all $\nu \in E_{0}$.

0.2. Definition. If $\delta \in \operatorname{Lim}\left(\omega_{1}\right)$, a ladder on $\delta$ is a strictly increasing function $\eta_{\delta}$ : $\omega \rightarrow \delta$ such that $\sup \left\{\eta_{\delta}(n): n \in \omega\right\}=\delta$. A ladder system on $E \subseteq \operatorname{Lim}\left(\omega_{1}\right)$ is a family $\left\{\eta_{\delta}: \delta \in E\right\}$ where each $\eta_{\delta}$ is a ladder on $\delta$.

If $\mathbf{P}$ is a partially ordered set (poset), and $p, q \in \mathbf{P}$, we say $p$ and $q$ are compatible if there is an $r \in \mathbf{P}$ such that $r \leqslant p$ and $r \leqslant q$. $\mathbf{P}$ is said to be c.c.c. (or satisfies the countable chain condition) if every uncountable subset of $\mathbf{P}$ contains a pair of compatible elements. A subset $D$ of $\mathbf{P}$ is called dense if for all $p \in \mathbf{P}$ there exist $q \in D$ such that $q \leqslant p$. Martin's Axiom (MA) is the statement that for every c.c.c. poset $\mathbf{P}$ and every $\lambda<2^{\boldsymbol{N}_{0}}$, if $\left\{D_{\nu}: \nu<\lambda\right\}$ is a family of dense subsets of $\mathbf{P}$, then there is a set $G \subseteq \mathbf{P}$ which is directed (i.e., for all $p, q \in G \exists r \in G$ s.t. $r \leqslant p, r \leqslant q$ ) such that for all $\nu \in \lambda, D_{\nu} \cap G \neq \varnothing ; \operatorname{MA}\left(\omega_{1}\right)$ is the preceding statement with $\lambda=\omega_{1}[\mathbf{J}$, p. 230].

0.3. THEOREM [ST]. If ZFC is consistent, then $\mathrm{ZFC}+\mathrm{MA}+\neg \mathrm{CH}$ is consistent.

A lemma which is often useful in verifying that a given $\mathbf{P}$ is c.c.c. is the following “ $\Delta$-lemma" [J, Lemma 22.6, p. 225].

0.4. LemMA. For any uncountable family $\mathcal{F}$ of finite sets there is an uncountable subfamily $\mathcal{F}^{\prime}$ and a finite set $\Delta$ such that $X \cap Y=\Delta$ for all $X, Y \in \mathcal{F}^{\prime}$.

I am grateful to S. Shelah for supplying the proof of the following in response to my query. (It is used to prove Theorem 2.8.) 
0.5. TheOREM (MA $\left.+{ }_{\neg} \mathrm{CH}\right)$. For any stationary set $E \subseteq \operatorname{Lim}\left(\omega_{1}\right)$ and any ladder system $\left\{\eta_{\delta}: \delta \in E\right\}$, there is a decomposition of $E$ into disjoint stationary sets, $E=\amalg_{\beta<\omega_{1}} E^{\beta}$, such that for all $\beta$ and all $\delta \in E^{\beta}, \delta>\beta$ and there are only finitely many $n \in \omega$ such that $\eta_{\delta}(n) \in E-E^{\beta}$.

Proof. We shall use MA $+\neg \mathrm{CH}$ to show that there exist functions $F_{n}: E \rightarrow \omega_{1}$ $(n \in \omega)$ such that

(i) $\forall n \in \omega \forall \delta \in E\left(\eta_{\delta}(n)<F_{n}(\delta)<\delta\right)$ and

(ii) $\forall n \in \omega \forall \delta \in E \exists N \forall m \geqslant N\left(\left(\eta_{\delta}(m) \in E\right) \Rightarrow F_{n}\left(\eta_{\delta}(m)\right)=F_{n}(\delta)\right)$.

Supposing for the moment that we can do this, define $S(n, \beta)=\left\{\delta \in E: F_{n}(\delta)=\right.$ $\beta\}$ for $n \in \omega, \beta \in \omega_{1}$. It suffices to prove that there is an $n$ such that there are uncountably many $\beta$ such that $S(n, \beta)$ is stationary (for the $S(n, \beta)$ will be disjoint for fixed $n$ ). Suppose, in order to obtain a contradiction, that there is no such $n$; then $\mu \stackrel{\text { def }}{=} \sup \{\beta: \exists n S(n, \beta)$ is stationary $\}$ is less than $\omega_{1}$. Hence, $E^{\prime} \stackrel{\text { def }}{=}\{\delta \in E$ : $\delta>\mu\}$ is stationary; since $E^{\prime}$ is the union of the sets $Y_{n} \stackrel{\text { def }}{=}\left\{\delta \in E^{\prime}: F_{n}(\delta)>\mu\right\}$ there is an $m$ such that $Y_{m}$ is stationary. Now $Y_{m}$ is the diagonal union of the sets $X_{\beta} \stackrel{\text { def }}{=} S(m, \beta) \cap Y_{m}$ for $\mu<\beta<\omega_{1}$, i.e., $Y_{m}=\left\{\delta: \exists \beta<\delta\left(\mu<\beta\right.\right.$ and $\left.\left.\delta \in X_{\beta}\right)\right\}$. But this is a contradiction, since each $S(m, \beta)$ is nonstationary for $\beta>\mu$, and the diagonal union of nonstationary sets is nonstationary (cf. [J, p. 58]).

Thus it remains to prove the existence of the functions $F_{n}$. Let us say that a finite set

$$
S=\left\{\left(n_{j}, \delta_{j}, N_{j}, \alpha_{j}\right) \in \omega \times E \times \omega \times \omega_{1}: j \leqslant k\right\}
$$

of 4-tuples is a condition if there exist functions $F_{n}: E \rightarrow \omega_{1}(n \in \omega)$ such that for all $j \leqslant k, \quad F_{n_{j}}\left(\delta_{j}\right)=\alpha_{j} ; \quad \eta_{\delta_{j}}\left(n_{j}\right)<\alpha_{j}<\delta_{j}$, and for $m \geqslant N_{j}$, if $\eta_{\delta_{j}}(m) \in E$, then $F_{n_{j}}\left(\eta_{\delta_{j}}(m)\right)=F_{n_{j}}\left(\delta_{j}\right)$. In an abuse of language, we shall say of $(n, \delta) \in \omega \times E$ that $(n, \delta) \in S$ if $\exists N, \alpha$ such that $(n, \delta, N, \alpha) \in S$; say $\delta \in S$ if $\exists n$ s.t. $(n, \delta) \in S$.

Let $\mathbf{P}$ consist of all conditions, partially ordered by $\supseteq$. Then one may check that for all $(n, \delta) \in \omega \times E, D_{(n, \delta)}=\{S \in \mathbf{P}:(n, \delta) \in S\}$ is dense in $\mathbf{P}$, and, by a standard argument, if $\mathbf{P}$ is c.c.c., $\mathrm{MA}+{ }_{\neg} \mathrm{CH}$ implies the existence of the desired family of functions.

So it remains to prove that $\mathbf{P}$ is c.c.c. Let $\left\{S_{\nu}: \nu<\omega_{1}\right\}$ be an uncountable subset of $\mathbf{P}$, where say $S_{\nu}=\left\{\left(n_{j}^{\nu}, \delta_{j}^{\nu}, N_{j}^{\delta}, \alpha_{j}^{\nu}\right): j \leqslant k_{\nu}\right\}$ and $\left\{F_{n}^{\nu}: n \in \omega\right\}$ is a family of functions: $E \rightarrow \omega_{1}$ showing that $S_{\nu}$ is a condition. Using the $\Delta$-lemma (0.4) we can assume that there is a finite set $\Delta$ such that for all $\nu<\omega_{1}, S_{\nu}=\Delta \cup S_{\nu}^{\prime}$ where if $\mu \neq \nu$, there is no $\delta$ s.t. $\delta \in S_{\mu}^{\prime}$ and $\delta \in S_{\nu}^{\prime}$. Moreover, without loss of generality (by restricting to an uncountable subset of $\left\{S_{\nu}: \nu<\omega_{1}\right\}$ ), there exist $n_{n}, N_{j}, k$ such that for all $\nu \in \omega_{1}, k_{\nu}=k$ and for all $j \leqslant k, n_{j}^{\nu}=n_{j}$ and $N_{j}^{\nu}=N_{j}$. Also without loss of generality for all $j \leqslant k, \delta_{j}^{\nu}>\nu$. Define for each $j \leqslant k$ a function $\varphi_{j}: E \rightarrow \omega_{1}$ by

$$
\varphi_{j}(\nu)=\max \left\{\eta_{\delta_{j}^{\nu}}(m): m \in \omega, \eta_{\delta_{j}^{\nu}}(m)<\nu\right\} .
$$

The $\varphi_{j}$ are all regressive functions, so by repeated use of Fodor's Theorem, there is a stationary subset $E^{\prime}$ of $E$ and a $\gamma<\omega_{1}$ such that, for all $j \leqslant k$ and all $\nu \in E^{\prime}$, 
$\varphi_{j}(\nu)<\gamma$. Now (by restricting to an uncountable subset), we may assume that, for all $\mu, \nu<\omega_{1}$ and all $j \leqslant k$, the ladders $\eta_{\delta_{j}^{\nu}}$ and $\eta_{\delta_{j}^{\mu}}$ are identical below $\gamma$. Moreover, we may assume that if there is an $m \geqslant N_{j}$ such that $\eta_{\delta_{j}^{\nu}}(m) \in E \cap \nu$ for some (hence all) $\nu$, then for all $v, \mu<\omega_{1}, \alpha_{j}^{\nu}=\alpha_{j}^{\mu}$ (since in this case $\alpha_{j}^{\nu}=F_{n_{j}}\left(\eta_{\delta_{j}^{\nu}}(m)\right)<\eta_{\delta_{j}^{\nu}}(m)$ $<\gamma$ ). Then if we pick $\nu<\mu$ such that for all $j \leqslant k, \delta_{j}^{\nu}<\mu$, one may prove that $S_{\mu} \cup S_{\nu}$ is a condition by defining the function $F_{n_{j}}$ to be $F_{n_{j}}^{\nu}$ on $[0, \mu\rangle$ and $F_{n_{j}}^{\mu}$ on $\left[\mu, \omega_{1}\right\rangle$.

The remainder of this section will assume some familiarity with the method of forcing. This material is used only for the proofs of 2.10, 2.11, 3.4 and 3.8, and, even there, knowledge of the details of proofs of 0.7 and 0.8 is not needed. The following notions are due to Shelah. (See [B2, D, H or S2] for details.)

If $A$ is any set, $\mathscr{P}(A)$ (resp. $\mathscr{P}_{\omega_{1}}(A)$ ) denotes the set of all (resp. all countable) subsets of $A$. A subset $\mathcal{S} \subseteq \mathscr{P}_{\omega_{1}}(A)$ is called a $c u b$ if it is closed under unions of countable chains and if for all $X \in \mathscr{P}_{\omega_{1}}(A)$ there exists $Y \in \mathcal{S}$ such that $X \subseteq Y$. Let $\mathbf{P}$ be a poset. If $q \in \mathbf{P}$ and $N \in \mathcal{P}_{\omega_{1}}(\mathbf{P} \cup \mathcal{P}(\mathbf{P})), q$ is said to be $(\mathbf{P}, N)$-generic if for every $D \in N$ such that $D$ is a dense subset of $\mathbf{P}$, and for every $r \leqslant q$, there exists $p \in D \cap N$ such that $p$ and $r$ are compatible. $\mathbf{P}$ is said to be proper if there is a cub $\mathcal{S}$ in $\mathscr{P}_{\omega_{1}}(\mathbf{P} \cup \mathcal{P}(\mathbf{P}))$ such that for all $N \in \delta$ and all $p \in N$ there is a $q \leqslant p$ such that $q$ is $(\mathbf{P}, N)$-generic.

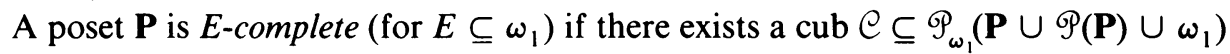
satisfying for all $N \in \mathcal{C}$, if $N \cap \omega_{1} \in E$ and $\left\{p_{n}: n \in \omega\right\} \subseteq \mathbf{P} \cap N$ such that (i) $p_{n+1} \leqslant p_{n}$ for all $n$, and (ii) for all dense subsets $D$ of $\mathbf{P}$ which belong to $N$, there is an $n \in \omega$ such that $p_{n} \in D$; then there is a $q \in \mathbf{P}$ such that for all $n \in \omega, q \leqslant p_{n}$.

0.6. Definition. We shall make use of the following hypotheses ('PFA' stands for Proper Forcing Axiom).

$\operatorname{PFA}(\lambda)$ : if $\mathbf{P}$ is a proper poset of cardinality $\leqslant \lambda$ and $\left\{D_{\nu}: \nu<\omega_{1}\right\}$ is a family of dense subsets of $\mathbf{P}$, then there is a directed subset $G \subset \mathbf{P}$ such that for all $\nu<\omega_{1}$, $D_{\nu} \cap G \neq \varnothing$.

PFA: for all cardinals $\lambda, \operatorname{PFA}(\lambda)$.

$+\left(\omega_{1}-S\right)$ : there is a stationary and costationary subset $S$ of $\omega_{1}$ such that: (i) if $\mathrm{P}$ is a proper poset of cardinality $\omega_{1}$ which is $\left(\omega_{1}-S\right)$-complete, and $\left\{D_{\nu}: \nu<\omega_{1}\right\}$ is a family of dense subsets of $\mathbf{P}$, then there is a directed $G \subseteq \mathbf{P}$ such that for all $\nu<\omega_{1}, D_{\nu} \cap G \neq \varnothing$; and (ii) for all $E \subseteq \omega_{1}$ such that $\tilde{E} \nsubseteq \tilde{S}$ (i.e., $E \cap\left(\omega_{1}-S\right)$ is stationary), $\diamond(E)$ holds.

0.7. TheOREM (ShelaH). (1) ZFC $+P F A\left(\omega_{1}\right)$ implies $Z F C+M A\left(\omega_{1}\right)$.

(2) If $Z F C$ is consistent, then $Z F C+P F A\left(\omega_{1}\right)$ is consistent.

(3) If ZFC $+" \exists$ supercompact cardinal" is consistent, then $Z F C+P F A$ is consistent.

(4) If ZFC is consistent, so is $Z F C+G C H++\left(\omega_{1}-S\right)$.

For a proof see [B2, D, H, or S2]. See also [M2] for an exposition of an application to Whitehead's Problem.

From now on, we assume the consistency of ZFC. 
0.8. TheOREM. (1) There is a model of $\mathrm{ZFC}+\mathrm{MA}+\neg_{\mathrm{CH}}$ in which there are disjoint stationary subsets $E_{0}$ and $E_{1}$ of $\operatorname{Lim}\left(\omega_{1}\right)$ and a ladder system $\left\{\eta_{\delta}: \delta \in E_{0}\right\}$ such that

for every cub $\mathcal{C}$, there exists $\delta \in E_{0}$ such that for arbitrarily large $n \in \omega, \eta_{\delta}(n) \in \circlearrowright \cap E_{1}$.

(2) There is a model of $\mathrm{ZFC}+\mathrm{MA}+\neg \mathrm{CH}$ such that for any stationary $E \subseteq \operatorname{Lim}\left(\omega_{1}\right)$ and any ladder system $\left\{\eta_{\delta}\right.$ :

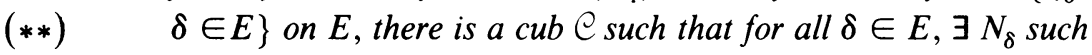
that for $n \geqslant N_{\delta}, \eta_{\delta}(n) \notin \bigodot$.

Proof. (1) It is consistent to assume that the universe is $L$.

Let $E_{0}$ and $E_{1}$ be any disjoint stationary subsets of $\operatorname{Lim}\left(\omega_{1}\right)$. By $\diamond\left(E_{0}\right)$ there is a sequence $\left\{S_{\delta}: \delta \in E_{0}\right\}$ such that for all $X \subseteq \omega_{1},\left\{\delta \in E_{0}: X \cap \delta=S_{\delta}\right\}$ is stationary (cf. [E, p. 21]). If $S_{\delta} \cap E_{1}$ is not cofinal in $\delta$, let $\eta_{\delta}: \omega \rightarrow \delta$ be any ladder on $\delta$. If $S_{\delta} \cap E_{1}$ is cofinal in $\delta$ define $\eta_{\delta}: \omega \rightarrow \delta$ so that its range is a cofinal subset of $S_{\delta} \cap E_{1}$. Now given any cub $\bigodot$ in $\omega_{1}$ let $\overline{\bigodot \cap E_{1}}$ denote the closure of $\bigodot \cap E_{1}$; this is a cub so there exists $\delta \in \overline{\left(\bigodot \cap E_{1}\right)} \cap E_{0}$ such that $\bigodot \cap \delta=S_{\delta}$. But then $S_{\delta} \cap E_{1}$ is cofinal in $\delta$ and by construction for all $n, \eta_{\delta}(n) \in S_{\delta} \cap E_{1}=\bigodot \cap E_{1}$. Thus we have proved that in $V,(*)$ holds. Now there is a c.c.c. poset $\mathbf{P}$ such that $V^{\mathbf{P}}$ ₹ ZFC $+\mathrm{MA}$ $+\neg \mathrm{CH}$ (see e.g. $[\mathbf{J}, \S 23])$. Since $\mathbf{P}$ is c.c.c.,

$$
V^{\mathbf{P}} \vDash \text { " } E_{0} \text { and } E_{1} \text { are stationary subsets of } \omega_{1} \text { " }
$$

(see e.g. [D, Lemma 2.1]). Also, since $\mathbf{P}$ is c.c.c., for every name $\tau$ such that

$$
V^{\mathbf{P}} F^{\text {" }} \tau \text { is a cub in } \omega_{1} \text { ", }
$$

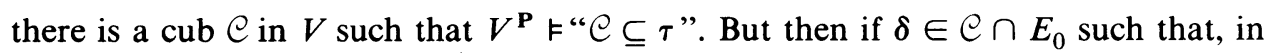
$V, \forall n \eta_{\delta}(n) \in \mathcal{C} \cap E_{1}$, the same holds in $V^{\mathbf{P}}$. Hence (*) holds in $V^{\mathbf{P}}$.

(2) We shall show that in a model of PFA, (**) holds. So let $\left\{\eta_{\delta}: \delta \in E\right\}$ be a ladder system on a stationary set $E \subseteq \operatorname{Lim}\left(\omega_{1}\right)$. Let $\mathbf{P}=\{C: C$ is a closed countable subset of $\omega_{1}$ s.t. for $\left.\delta \in E \exists N_{\delta} \forall n \geqslant N_{\delta}\left(\eta_{\delta}(n) \notin C\right)\right\}$ partially ordered by the relation of end extension, i.e., $C_{2} \leqslant C_{1}$ iff $C_{2} \cap\left(\sup C_{1}\right)=C_{1}$. For $\mu \in \omega_{1}$, let $D^{\mu}=\{C \in \mathbf{P}: \sup C>\mu\}$; then $D^{\mu}$ is dense in $\mathbf{P}$ since for any $C \in \mathbf{P}$, if $\mu \geqslant \sup C$, $C \cup\{\mu+1\} \in D_{\mu}$. If $\mathbf{P}$ is a proper poset, PFA says that there is a pairwise compatible subset $G$ of $\mathbf{P}$ such that $\forall \mu\left(G \cap D^{\mu} \neq \varnothing\right)$; then $\circlearrowright=\cup G$ is the desired cub. So it remains to prove $\mathbf{P}$ is proper; let $\delta$ be the cub in $\mathscr{P}_{\omega_{1}}(\mathbf{P} \cup \mathcal{P}(\mathbf{P}))$ consisting of sets $N=\mathscr{E} \amalg \mathscr{D}$ satisfying the following, where $\& \in \mathscr{P}_{\omega_{1}}(\mathbf{P}), \mathscr{D} \in \mathscr{P}_{\omega_{1}}(\mathscr{P}(\mathbf{P}))$ and $\sup N \stackrel{\text { def }}{=} \sup \&=\sup (\mathbf{P} \cap N)$ :

(a) for all $D \in \mathfrak{D}, D$ is a dense subset of $\mathbf{P}$, and $D \cap N$ is a dense subset of $\mathbf{P} \cap N$,

(b) for all $\mu<\omega_{1}, D^{\mu} \in \mathfrak{D} \Leftrightarrow \mu<\sup N$,

(c) if $C \in \mathbb{E}$ and $\nu<\sup N$, then $C \cup\{\nu\} \in \mathbf{P} \cap N$.

Let $\mathcal{S}^{*}=\left\{N \in \mathcal{\delta}: N=\cup_{n \in \omega} N_{n}\right.$, where for all $n, N_{n} \in \mathcal{\delta}, N_{n} \subseteq N_{n+1}$, and $\sup N_{n}<$ $\left.\sup N_{n+1}\right\}$. Clearly, $\mathcal{S}^{*}$ is a cub, so it suffices to prove that if $C \in N \in \mathcal{S}^{*}$, then there exists $C^{\prime} \in \mathbf{P}$ such that $C^{\prime} \leqslant C$ and for all $D \in \mathfrak{D} \in N, C^{\prime} \in D$; then $C^{\prime}$ is clearly $(\mathbf{P}, N)$-generic. 
Write $N=\cup_{n \in \omega} N_{n}$ as in the definition of $\delta^{*}$, and let $\sigma_{n}=\sup N_{n}$; thus $\left\{\sigma_{n}\right.$ : $n \in \omega\}$ is a strictly increasing sequence whose limit is $\sup N(=\delta$, say). Without loss of generality we may suppose that $C \in N_{0}$. If $N=\& I D D$, let $\left\{D_{n}: n \in \omega\right\}$ be an enumeration of $\mathfrak{D}$, where we may suppose that for all $n, D_{n} \in N_{n+1}$. We shall inductively define a chain

$$
C_{0} \geqslant C_{1} \geqslant \cdots \geqslant C_{n} \geqslant \cdots
$$

of elements of \& such that $C_{0}=C$, and for all $n \in \omega$, sup $C_{n+1}>\sigma_{n}$, and $C_{n+1} \in D_{n}$ $\cap N_{n+1}$. We shall then let $C^{\prime}=\cup_{n \in \omega} C_{n} \cup\{\delta\}$; in order to insure that $C^{\prime} \in \mathbf{P}$, we shall also require that if $\delta \in E$, then for all $n, m \in \omega, \eta_{\delta}(m) \in C_{n}$ implies $\eta_{\delta}(m)<\sigma_{0}$. Suppose $C_{n}$ has been chosen. Pick

$$
\nu_{n}>\max \left(\left\{\eta_{\delta}(k): k \in \omega, \eta_{\delta}(k)<\sigma_{n+1}\right\} \cup\left\{\sigma_{n}\right\}\right),
$$

and let $\tilde{C}_{n}=C_{n} \cup\left\{\nu_{n}\right\}$, which belongs to $\mathbf{P} \cap N_{n+1}$ by (c). Then by (a) there exists $C_{n+1} \in \mathbf{P} \cap N_{n+1}$ such that $\tilde{C}_{n} \geqslant C_{n+1}$ and $C_{n+1} \in D_{n}$.

By $0.7(1)$ and (3) the preceding argument shows the existence of a model of $(* *)$, assuming the existence of a model of ZFC with a supercompact cardinal. However, the latter assumption can be eliminated. (I am grateful to $\mathrm{M}$. Magidor for the following argument.) Notice that $|\mathbf{P}|=\boldsymbol{\aleph}_{1}$ provided $\mathrm{CH}$ holds. We shall make use of the following lemma.

0.9. Lemma. If $V \vDash C H$ and $\mathbf{P}$ is a proper poset of cardinality $\boldsymbol{\aleph}_{1}$, then $V^{\mathbf{P}} \vDash C H$.

Assuming the lemma, we can, by standard methods of iterated forcing (cf. [B1, J or $\mathbf{H}]$ ), construct an iteration sequence $\left(\mathbf{P}_{i}\right)_{i<\omega_{2}}$ with countable support such that for all $i<\omega_{2}, \mathbf{P}_{i+1}=\mathbf{P}_{i} * Q_{i}$ where $V^{\mathbf{P}_{i}} \vDash\left(\mathrm{CH}^{*}\right.$ " $Q_{i}$ is proper and of cardinality $\boldsymbol{\aleph}_{1}$ "), and such that if $\mathbf{P}=\underset{\rightarrow}{\rightarrow}\left(\mathbf{P}_{i}\right)_{i<\omega_{2}}$, then $V^{\mathbf{P}} \vDash(* *)$. (Since $\mathbf{P}$ is proper, $\omega_{1}$ does not collapse.)

Proof of 0.9. It suffices to prove that if $p \in \mathbf{P}$ and $\tau$ is a name such that $p \Vdash$ “ $\tau \subseteq \check{\omega}$ ", then for any generic $G$ containing $p$ there is a countable subset $N^{\prime} \subseteq \mathbf{P}$ such that for all $n \in \omega, V[G] \vDash$ " $n \in \tau$ " iff $\exists p^{\prime} \in N^{\prime} \cap G, p^{\prime} \leqslant p$, such that $p^{\prime} \Vdash^{\prime \prime} \check{n} \in \tau$ ”. But $D=\{q \leqslant p: \exists$ countable submodel $N$ of the universe s.t. $p, \tau \in N$ and $q$ is $(\mathbf{P}, N)$-generic $\}$ is dense below $p$, since $\mathbf{P}$ is proper; so if $p \in G, \exists q \in D \cap G$. If $N$ is as in the definition of $D$ for this $q$, let $N^{\prime}=\mathbf{P} \cap N$. Then for all $n \in \omega$, $D_{n}^{\prime}=\left\{p^{\prime} \leqslant p: p^{\prime} \mathbb{H}^{\prime \prime} \check{n} \in \tau^{\prime \prime}\right\}$ is dense below $p$ and belongs to $N$ so since $q$ is $(\mathbf{P}, N)$-generic, $\exists p^{\prime} \in D_{n}^{\prime} \cap N^{\prime} \cap G$.

An alternate proof of $0.8(2)$ can be given using 0.7(2): see the article by Mekler in the Proceedings of the Honolulu Conference on Abelian Groups, Springer-Verlag Lecture Notes in Mathematics.

1. The classification theorem. Throughout the rest of this paper we shall be considering the structure and classification of strongly $\omega_{1}$-free abelian groups of cardinality $\omega_{1}$. A group $A$ is strongly $\omega_{1}$-free if it is $\omega_{1}$-free and every countable subgroup of $A$ is contained in a countable subgroup $B$ such that $A / B$ is $\omega_{1}$-free. (We say $B$ is $\omega_{1}$-pure in $A$.) Mekler [M1, Theorem 25] has shown that MA $+\neg \mathrm{CH}$ implies that the strongly $\omega_{1}$-free groups of cardinality $\omega_{1}$ are precisely the $\omega_{1}$-separable groups (but this is not the case in a model of $\mathrm{CH}$ [S3]). 
A principal technique in the study of strongly $\omega_{1}$-free groups is the use of $\omega_{1}$-filtrations (cf. [E, Chapter 2]). If $A$ is $\omega_{1}$-free of cardinality $\omega_{1}, A$ has an $\omega_{1}$-filtration i.e., a chain $\left\{A_{\nu}: \nu<\omega_{1}\right\}$ of countable subgroups of $A$ such that $A=\cup_{\nu<\omega_{1}} A_{\nu}$ and for all limit ordinals $\delta<\omega_{1}, A_{\delta}=\cup_{\nu<\delta} A_{\nu}$. Moreover, if $A$ is strongly $\omega_{1}$-free, $A$ has an $\omega_{1}$-filtration which satisfies, in addition,

(i) $A / A_{0}$ is $\omega_{1}$-free, and for all $\nu<\omega_{1}, A / A_{\nu+1}$ is $\omega_{1}$-free, and

(ii) for all $\nu<\omega_{1}, A_{\nu+1} / A_{\nu} \cong A_{\nu+1} / A_{\nu} \oplus \mathbf{Z}^{(\omega)}$.

(Note that if (i) holds, then for all $\nu$, for sufficiently large $\tau>\nu, A_{\tau} / A_{\nu+1} \cong \mathbf{Z}^{(\omega)}$.)

From now on, whenever we write $A=\cup_{\nu<\omega_{1}} A_{\nu}$ we shall mean that $\left\{A_{\nu}: \nu<\omega_{1}\right\}$ is an $\omega_{1}$-filtration of $A$ satisfying (i) and (ii) above; $\omega_{1}$-filtrations of $A$ agree on a cub (cf. [E, p. 26]):

1.1. LEMMA. If $\left\{A_{\nu}: \nu<\omega_{1}\right\}$ and $\left\{A_{\nu}^{\prime}: \nu<\omega_{1}\right\}$ are both $\omega_{1}$-filtrations, then there is a cub $\mathcal{C}$ in $\omega_{1}$ such that for all $\nu \in \mathcal{C}, A_{\nu}=A_{\nu}^{\prime}$.

It follows that we can associate to $A$ a given equivalence class of subsets of $\omega_{1}$, which is an invariant of $A$. In fact, if $A=\cup_{\nu<\omega_{1}} A_{\nu}$, let $E=\left\{\delta<\omega_{1}: A / A_{\delta}\right.$ is not $\omega_{1}$-free $\}$; by property (i) above, $E \subseteq \operatorname{Lim}\left(\omega_{1}\right)$. By Lemma 1.1 above $E$ is uniquely determined by $A$ "up to a cub," i.e., if we let $\Gamma(A)=\tilde{E} \stackrel{\text { def }}{=}\left\{E^{\prime} \subseteq \omega_{1}: \exists\right.$ cub $\bigodot$ s.t. $\left.E \cap \mathcal{C}=E^{\prime} \cap \mathcal{C}\right\}$, then $\Gamma(A)$ is independent of the choice of $\omega_{1}$-filtration of $A$.

Let $D\left(\omega_{1}\right)=\left\{\tilde{E}: E \subseteq \omega_{1}\right\}$; it is a Boolean algebra under the ordering induced by $\subseteq$. If $\left\{\tilde{E}_{\nu}: \nu<\omega_{1}\right\} \subseteq D\left(\omega_{1}\right)$, then the sup of this set, denoted $\vee\left\{\tilde{E}_{\nu}: \nu<\omega_{1}\right\}$, equals the equivalence class of $\cup_{\nu<\omega_{1}}\left(E_{\nu}-(\nu+1)\right)$. (Use [J, Lemma 7.5].) If $A=\bigoplus_{\nu<\omega_{1}} A_{\nu}$, then $\Gamma(A)=\bigvee\left\{\Gamma\left(A_{\nu}\right): \nu<\omega_{1}\right\}$. Note also that the sup of $\left\{\tilde{E}_{n}\right.$ : $n<\omega\}$ is $\left(\cup_{n} E_{n}\right)$.

Definition. If $A$ and $B$ are strongly $\omega_{1}$-free groups, $A$ and $B$ are filtration-equivalent (denoted $A \approx B$ ), if there are $\omega_{1}$-filtrations $A=\cup_{\nu<\omega_{1}} A_{\nu}$ and $B=\cup_{\nu<\omega_{1}} B_{\nu}$ such that for all $\nu<\omega_{1}$ there is an isomorphism $f_{\nu}: A_{\nu} \rightarrow B_{\nu}$ such that for all $\mu<\nu$, $f_{\nu}\left(A_{\mu}\right)=B_{\mu}$. We shall call such an $f_{\nu}$ a level-preserving (l.p.) isomorphism (from $A_{\nu}$ onto $B_{\nu}$ ).

It is easy to see that if $A$ and $B$ are filtration-equivalent then they are quotientequivalent, i.e., there are $\omega_{1}$-filtrations $A=\cup_{\nu<\omega_{1}} A_{\nu}, B=\cup_{\nu<\omega_{1}} B_{\nu}$ such that for all $\nu<\mu, A_{\mu} / A_{\nu} \cong B_{\mu} / B_{\nu}$; so, in particular, $\Gamma(A)=\Gamma(B)$. It is known that there exist quotient-equivalent groups which are nonisomorphic [E or EMS]. However, we have the following theorem of $\mathrm{ZFC}+\mathrm{MA}+{ }_{\neg} \mathrm{CH}$ (which is not a theorem of $\mathrm{ZFC}+\mathrm{CH}$ by Theorem 3.2; but see also 3.4).

1.2. THEOREM (MA $+\neg \mathrm{CH})$. If $A$ and $B$ are $\omega_{1}$-separable groups of cardinality $\omega_{1}$ which are filtration-equivalent, then they are isomorphic.

Proof. Fix $\omega_{1}$-filtrations $A=\cup_{\nu<\omega_{1}} A_{\nu}$ and $B=\cup_{\nu<\omega_{1}} B_{\nu}$ such that for every $\nu<\omega_{1}$ there is a level-preserving isomorphism from $A_{\nu}$ onto $B_{\nu}$. Let $\mathbf{P}$ be the set of all isomorphisms $\varphi: L \rightarrow L^{\prime}$ where $\varphi$ is the restriction of some 1.p. isomorphism and $L$ (resp. $L^{\prime}$ ) is a finitely-generated pure subgroup of $A$ (resp. $B$ ). Partially order $\mathbf{P}$ by 
$\supseteq$. For each $a \in A$ (resp. $b \in B$ ) let $D_{a}=\{\varphi \in \mathbf{P}: a \in \operatorname{Dom} \varphi\}\left(D_{b}=\{\varphi \in \mathbf{P}\right.$ : $b \in \operatorname{Ran} \varphi\}$ ). We claim that $D_{a}$ (and similarly $D_{b}$ ) is dense in $\mathbf{P}$. Indeed, let $a \in A$, $\varphi \in \mathbf{P}$; say $\varphi: L \rightarrow L^{\prime}$ is the restriction of some level-preserving isomorphism $f$ : $A_{\nu} \rightarrow B_{\nu}$; since $L$ is finitely-generated we may assume that $\nu=\sigma+1$ for some $\sigma<\omega_{1}$. Choose $\tau \geqslant \nu$ such that $a \in A_{\tau}$, and let $g: A_{\tau} \rightarrow B_{\tau}$ be a level-preserving isomorphism (which exists because $A$ and $B$ are filtration-equivalent). Then since $A / A_{\nu}\left(=A / A_{\sigma+1}\right)$ is $\omega_{1}$-free (by property (i) of an $\omega_{1}$-filtration), we have $A_{\tau}=A_{\nu} \oplus$ $F$ for some free group $F$, and then $B_{\tau}=B_{\nu} \oplus g(F)$; so if we define $h: A_{\tau} \rightarrow B_{\tau}$ by $h \uparrow A_{\nu}=f, h \uparrow F=g$, then $h$ is a level-preserving isomorphism. (Note that if we define for $a \in A, l(a)=$ least $\mu$ s.t. $a \in A_{\mu}$, then if $a=x+y, l(a)=$ $\max \{l(x), l(y)\}$ if $l(x) \neq l(y))$. If we let $L_{1}=\langle L, a\rangle_{*}, L_{1}^{\prime}=\left\langle L^{\prime}, h(a)\right\rangle_{*}$ and $\varphi_{1}=h \uparrow L_{1}$ then $\varphi_{1} \in D_{a}$ and $\varphi_{1} \leqslant \varphi$.

Since $D_{a}$ and $D_{b}$ are dense for all $a \in A, b \in B$, if $\boldsymbol{\aleph}_{1}<2^{\boldsymbol{\aleph}_{0}}$ and $\mathbf{P}$ is c.c.c., MA implies there exists a directed $G \subseteq \mathbf{P}$ which intersects every $D_{a}$ and $D_{b}$; then $\cup G$ is an isomorphism of $A$ onto $B$. Thus it remains to prove that $\mathbf{P}$ is c.c.c. (The following argument is an improvement on my original proof which owes much to one found by Alan Mekler.)

Let $S$ be an uncountable subset of $\mathbf{P}$. As in [E, p. 68], we can assume that there is a finitely generated pure subgroup $T$ of $A$ such that for all $\varphi \neq \Psi$ in $\mathcal{S}$, Dom $\varphi \cap$ Dom $\Psi=T$ and $\varphi \uparrow T=\Psi \uparrow T$. Also, without loss of generality, $T \subseteq A_{0}$. Construct by induction a sequence $\left\{\varphi_{\nu}: \nu<\omega_{1}\right\}$ of elements of $\mathcal{S}$ such that if $D_{\nu} \stackrel{\text { def }}{=} \operatorname{Dom} \varphi_{\nu}$, $D_{\nu} \cap A_{\nu+1}=T$; it follows that

$$
\left\langle D_{\nu}+A_{\nu}\right\rangle_{*} \cap A_{\nu+1}=A_{\nu}
$$

and therefore $\left\langle D_{\nu}+A_{\nu}\right\rangle_{*} / A_{\nu}$ is free (cf. [E, proof of 7.1]). Say $\varphi_{\nu}$ is a restriction of the l.p. isomorphism $g_{\nu}: A_{\sigma_{\nu}+1} \rightarrow B_{\sigma_{\nu}+1}$.

For each $\nu \in \operatorname{Lim}\left(\omega_{1}\right)$, let $\theta(\nu)=$ the least $\gamma<\nu$ such that there is a basis $x_{0}^{\nu}, \ldots, x_{m_{\nu}}^{\nu}$ of $D_{\nu}$ and representatives $y_{0}^{\nu}, \ldots, y_{n_{\nu}}^{\nu}$ of a basis of $\left\langle D_{\nu}+A_{\nu}\right\rangle_{*} / A_{\nu}$ such that each $x_{i}(0 \leqslant i \leqslant m)$ is a linear combination of the $y_{j}^{\nu}$ 's modulo $A_{\gamma}$. By Fodor's Theorem $(0.1)$, there is a stationary $E_{0} \subseteq \operatorname{Lim}\left(\omega_{1}\right)$ and a $\gamma<\omega_{1}$ such that for all $\nu \in E_{0}, \theta(\nu)=\gamma$. By restricting to an uncountable subset of $E_{0}$ we can assume that there are $m, n \in \omega, d_{i j} \in \mathbf{Z}(i \leqslant m, j \leqslant n)$ and elements $w_{0}, \ldots, w_{m}$ of $A_{\gamma}$ such that for all $\nu \in E_{0}$, there is a basis $x_{0}^{\nu}, \ldots, x_{m}^{\nu}$ of $D_{\nu}$, and representatives $y_{0}^{\nu}, \ldots, y_{n}^{\nu}$ of a basis of $\left\langle D_{\nu}+A_{\nu}\right\rangle_{*} / A_{\nu}$ such that for all $i \leqslant m$,

$$
x_{i}^{\nu}=\sum_{j=0}^{n} d_{i j} y_{j}^{\nu}+w_{i} .
$$

Moreover, we can assume that, for all $\mu, \nu$ in $E_{0}$ and all $i \leqslant m, g_{\mu}\left(w_{i}\right)=g_{\nu}\left(w_{i}\right)$.

Now choose $\mu<\nu$ in $E_{0}$ such that $\sigma_{\mu}+1<\nu$ and write $A_{\sigma_{\nu}+1}=A_{\sigma_{\mu}+1} \oplus C$, where $y_{0}^{\nu}, \ldots, y_{n}^{\nu} \in C\left(\cong \mathbf{Z}^{(\omega)}\right)$. (This is possible because $y_{0}^{\nu}, \ldots, y_{n}^{\nu} \in\left\langle D_{\nu}+A_{\nu}\right\rangle_{*} \subseteq$ $A_{\sigma_{\nu}+1}$ and $\left\{y_{0}^{\nu}, \ldots, y_{n}^{\nu}\right\}$ is pure-independent $\bmod A_{\nu}$ and hence pure-independent $\left.\bmod A_{\sigma_{\mu}+1}\right)$. Define $h: A_{\sigma_{\nu}+1} \rightarrow B_{\sigma_{\nu}+1}$ to be $g_{\mu}$ on $A_{\sigma_{\mu}+1}$ and $g_{\nu}$ on $C$. Then $h$ is a l.p. 
isomorphism and $h \uparrow D_{\mu}=\varphi_{\mu}$. Also for any $x_{i}^{\nu}(i \leqslant m)$,

$$
\begin{aligned}
h\left(x_{\nu}^{i}\right) & =h\left(\sum_{j=0}^{n} d_{i j} y_{j}^{\nu}+w_{i}\right)=\sum_{j=0}^{n} d_{i j} g_{\nu}\left(y_{j}^{\nu}\right)+g_{\mu}\left(w_{i}\right) \\
& =\sum_{j=0}^{n} d_{i j} g_{\nu}\left(y_{j}^{\nu}\right)+g_{\nu}\left(w_{i}\right)=\varphi_{\nu}\left(x_{\nu}^{i}\right) .
\end{aligned}
$$

Thus $h \uparrow D_{\nu}=\varphi_{\nu}$, so $h \uparrow\left\langle D_{\mu}, D_{\nu}\right\rangle_{*}$ is an element of $\mathbf{P}$ extending both $\varphi_{\mu}$ and $\varphi_{\nu}$.

In the next section we shall present some applications of this theorem which give structural information about arbitrary $\omega_{1}$-separable groups. For the remainder of this section we shall show how the relation of filtration-equivalence can be given a more explicit meaning for some particularly simple quotient-equivalence classes of strongly $\omega_{1}$-free groups. This analysis is not needed for the results of $\S 2$, but the arguments used here to provide simple paradigms for some of the more complex ones which follow in the next section.

1.3. Definition. If $H$ is a countable torsion-free group which is not free and $A$ is a strongly $\omega_{1}$-free group of cardinality $\omega_{1}$, we shall say that $A$ is of type $H$ if $A$ has an $\omega_{1}$-filtration $A=\cup_{\nu<\omega_{1}} A_{\nu}$ such that whenever $A_{\nu}$ is not $\omega_{1}$-pure in $A$, then $A_{\nu+1} / A_{\nu} \cong H \oplus \mathbf{Z}^{(\omega)}$.

Suppose, for example, that $A=\cup_{\nu<\omega_{1}} A_{\nu}$ is of type $Q^{(p)}$ where $p$ is a prime and $Q^{(p)}$ is the group of rationals whose denominators are powers of $p$. If $E=\left\{\delta<\omega_{1}\right.$ : $A_{\delta}$ is not $\omega_{1}$-pure in $\left.A\right\}$, choose for each $\delta \in E$ an element $y_{\delta} \in A_{\delta+1}$ such that $\left\langle\left\{y_{\delta}\right\} \cup A_{\delta}\right\rangle_{*} / A_{\delta} \cong Q^{(p)}$. Let $\delta \in E$. We claim that there is a ladder $\eta_{\delta}$ on $\delta$ and a strictly increasing function $k_{\delta}: \omega \rightarrow \omega-\{0\}$ such that, for all $m \in \omega-\{0\}$, if $k_{\delta}(n-1)<m \leqslant k_{\delta}(n)$ (where $k_{\delta}(-1)=0$ ) then $p^{m}$ divides $y_{\delta} \bmod A_{\mu+1}$ iff $\mu \geqslant$ $\eta_{\delta}(n)$. Indeed, we define the functions by induction: $\eta_{\delta}(0)=$ least $\nu<\delta$ such that $p$ divides $y_{\delta} \bmod A_{\nu+1}$ and $k_{\delta}(0)=$ the largest $k$ such that $p^{k}$ divides $y_{\delta} \bmod A_{\eta_{\delta}(0)+1}$. (Note that $A_{\delta+1} / A_{\eta_{\delta}(0)+1}$ is free so $k_{\delta}(0)$ is defined.) If $\eta_{\delta}(n)$ and $k_{\delta}(n)$ have been defined, let $d=k_{\delta}(n)+1$ and let $\eta_{\delta}(n+1)=$ least $\nu<\delta$ such that $p^{d}$ divides $y_{\delta} \bmod A_{\nu+1}$, and let $k_{\delta}(n+1)=$ the largest $k$ such that $p^{k}$ divides $y_{\delta} \bmod A_{\eta_{\delta}(n+1)+1}$. (Again $\eta_{\delta}(n+1)$ exists since $A_{\delta+1} / A_{\eta_{\delta}(n+1)+1}$ is free.)

Now the set $E$ and the functions $\eta_{\delta}$ and $k_{\delta}$ are uniquely determined once the $\omega_{1}$-filtration $A=\cup_{\nu<\omega_{1}} A_{\nu}$ and the elements $y_{\delta}$ have been chosen. The function $\eta_{\delta}$ is called an associated ladder to $A$ at $\delta$. Define a function $\mathscr{D}$ on $E$ by $\mathscr{D}(\delta)=\left(\eta_{\delta}, k_{\delta}\right)$ for all $\delta \in E$, and call $\mathscr{D}$ an associated divisibility function for $A$.

1.4. THEOREM. If $A$ and $A^{\prime}$ are strongly $\omega_{1}$-free groups of type $Q^{(p)}$ which have $\omega_{1}$-filtrations with identical associated divisibility functions, then $A$ is filtration-equivalent to $A^{\prime}$.

Proof. By hypothesis there are $\omega_{1}$-filtrations $A=\cup_{\nu<\omega_{1}} A_{\nu}$ and $A^{\prime}=\cup_{\nu<\omega_{1}} A_{\nu}^{\prime}$, a set $E \subseteq \operatorname{Lim}\left(\omega_{1}\right)$ and elements $y_{\delta} \in A_{\delta+1}, y_{\delta}^{\prime} \in A_{\delta+1}^{\prime}$ such that

$$
\begin{aligned}
& E=\left\{\nu<\omega_{1}: A_{\nu} \text { is not } \omega_{1} \text {-pure in } A\right\} \\
& =\left\{\nu<\omega_{1}: A_{\nu}^{\prime} \text { is not } \omega_{1} \text {-pure in } A^{\prime}\right\} ; \\
& A_{\delta+1} / A_{\delta} \cong\left\langle\left\{y_{\delta}\right\} \cup A_{\delta}\right\rangle_{*} / A_{\delta} \oplus \mathbf{Z}^{(\omega)}, \\
& A_{\delta+1}^{\prime} / A_{\delta}^{\prime} \cong\left\langle\left\{y_{\delta}^{\prime}\right\} \cup A_{\delta}\right\rangle_{*} / A_{\delta}^{\prime} \oplus \mathbf{Z}^{(\omega)},
\end{aligned}
$$


and the corresponding $\mathscr{D}$ and $\mathscr{Q}^{\prime}$ are identical, i.e., for all $\delta \in E, \mu<\delta$ and $m \in \omega$,

$$
p^{m} \mid y_{\delta} \bmod A_{\mu+1} \text { iff } p^{m} \mid y_{\delta}^{\prime} \bmod A_{\mu+1}^{\prime} .
$$

To show that $A$ and $A^{\prime}$ are filtration-equivalent we shall prove by induction on $\nu$ the following stronger result:

for all $\mu<\nu<\omega_{1}$, given a l.p. isomorphism $f: A_{\mu+1} \rightarrow A_{\mu+1}^{\prime}$ and given $x$ (resp. $x^{\prime}$ ) in $A_{\nu+1}$ (resp. $A_{\nu+1}^{\prime}$ ) such that $x+A_{\nu}$ (resp. $x^{\prime}+A_{\nu}^{\prime}$ ) generates a free direct summand of $A_{\nu+1} / A_{\nu}$ (resp. $A_{\nu+1}^{\prime} / A_{\nu}^{\prime}$ ), there is a 1.p. isomorphism $\tilde{f}: A_{\nu+1} \rightarrow A_{\nu+1}^{\prime}$ extending $f$ such that $\tilde{f}(x)=x^{\prime}$.

The proof is by induction on $\nu$; there are 3 cases, 2 of them easy.

Case 1. $\nu=\tau+1$ for some $\tau$. By induction we may assume that $\tau=\mu$. Then since $A_{\nu+1} / A_{\mu+1}$ (resp. $A_{\nu+1}^{\prime} / A_{\mu+1}^{\prime}$ ) is free and

$$
\left\langle x+A_{\mu+1}\right\rangle / A_{\mu+1} \quad\left(\operatorname{resp} .\left\langle x^{\prime}+A_{\mu+1}^{\prime}\right\rangle /\left(A_{\mu+1}^{\prime}\right)\right)
$$

is a direct summand, it is clear that we can extend $f: A_{\mu+1} \rightarrow A_{\mu+1}^{\prime}$ to $\tilde{f}: A_{\nu+1} \rightarrow A_{\nu+1}^{\prime}$ s.t. $\tilde{f}(x)=x^{\prime}$.

Case 2. $\nu \in \operatorname{Lim}\left(\omega_{1}\right)-E$. Choose a strictly increasing sequence $\alpha_{n}$ approaching $\nu$ such that $\alpha_{0}>\mu$. Then by induction define a chain of 1.p. isomorphisms $g_{n}$ : $A_{\alpha_{n}+1} \rightarrow A_{\alpha_{n}+1}^{\prime}$ (with $g_{-1}=f$ ). If $g=\cup_{n \in \omega} g_{n}$, then $g$ is a 1.p. isomorphism: $A_{\nu} \rightarrow A_{\nu}^{\prime}$, and since $A_{\nu+1} / A_{\nu}$ is free we can extend to $\tilde{f}$ just as in Case 1.

Case 3. $\nu=\delta \in E$. We have

$$
\begin{aligned}
& A_{\delta+1} / A_{\delta}=\left\langle\left\{y_{\delta}\right\} \cup A_{\delta}\right\rangle_{*} / A_{\delta} \oplus \mathbf{Z}\left(x+A_{\delta}\right) / A_{\delta} \oplus F, \\
& A_{\delta+1}^{\prime} / A_{\delta}^{\prime}=\left\langle\left\{y_{\delta}^{\prime}\right\} \cup A_{\delta}^{\prime}\right\rangle_{*} / A_{\delta}^{\prime} \oplus \mathbf{Z}\left(x^{\prime}+A_{\delta}^{\prime}\right) / A_{\delta} \oplus F^{\prime},
\end{aligned}
$$

where $F$ and $F^{\prime}$ are free of rank $\omega$. Moreover, by hypothesis there is a ladder $\eta_{\delta}$ and a strictly increasing function $k_{\delta}: \omega \rightarrow \omega-\{0\}$ such that for all $m, n$ if $k_{\delta}(n-1)<$ $m \leqslant k_{\delta}(n)$ and $\alpha_{n}=\eta_{\delta}(n)$ then for all $\tau<\delta$,

$$
p^{m} \mid y_{\delta} \bmod A_{\tau+1} \quad \text { iff } \tau \geqslant \alpha_{n} \quad \text { iff } p^{m} \mid y_{\delta}^{\prime} \bmod A_{\tau+1}^{\prime} .
$$

Since $\delta$ is fixed write $k(n)$ for $k_{\delta}(n)$. By replacing $y_{\delta}$ (resp. $y_{\delta}^{\prime}$ ) by $y_{\delta}+u$ (resp. $y_{\delta}+u^{\prime}$ ) for suitable $u \in A_{\mu+2}$ (resp. $u^{\prime} \in A_{\mu+2}^{\prime}$ ) we can assume that $\alpha_{0}>\mu$. We shall define by induction on $n$ a chain of 1.p. isomorphisms $g_{n}: A_{\alpha_{n}+1} \rightarrow A_{\alpha_{n}+1}^{\prime}$ each extending $f$ and such that there exists $a_{n} \in A_{\alpha_{n}+1}$ such that $p^{k(n)} \mid y_{\delta}-a_{n}$ and $p^{k(n)} \mid y_{\mu}^{\prime}-g_{n}\left(a_{n}\right)$ in $A^{\prime}$.

Suppose for the moment that we can do this. Let

$$
z_{n}=\frac{y_{\delta}-a_{n}}{p^{k(n)}}, \quad z_{n}^{\prime}=\frac{y_{\delta}^{\prime}-g_{n}\left(a_{n}\right)}{p^{k(n)}}
$$

and notice that

$$
\left\langle\left\{y_{\delta}\right\} \cup A_{\delta}\right\rangle_{*}=\left\langle\left\{z_{n}: n \in \omega\right\} \cup A_{\delta}\right\rangle
$$

and similarly for $\left\langle\left\{y_{\delta}^{\prime}\right\} \cup A_{\delta}^{\prime}\right\rangle_{*}$. Now

$$
\bigcup_{n \in \omega} g_{n}: A_{\delta} \rightarrow A_{\delta}^{\prime}
$$


is a l.p. isomorphism which we can extend to $\tilde{f}: A_{\delta+1} \rightarrow A_{\delta+1}^{\prime}$ by sending representatives of a basis of $F$ onto representatives of a basis of $F^{\prime}$ and defining $\tilde{f}(x)=x^{\prime}$, and for all $n, \tilde{f}\left(z_{n}\right)=z_{n}^{\prime}$.

So it remains to define the $g_{n}$ 's. Suppose $g_{n}: A_{\alpha_{n}+1} \rightarrow A_{\alpha_{n}+1}^{\prime}$ and $a_{0}, \ldots, a_{n}$ have been defined for some $n \geqslant-1$ (where we let $g_{-1}=f, \alpha_{-1}=\mu$ ). Since $n$ is fixed let us write $\alpha$ for $\alpha_{n+1}, k$ for $k(n)$ and $k+d$ for $k(n+1)$. Now there exists $w \in A_{\alpha+1}$ such that $p^{k+d} \mid y_{\delta}-w$ (by definition of $\alpha_{n+1}$ and $k(n+1)$ ). Moreover, since $p^{k} \mid y_{\delta}-a_{n}$, we have $p^{k} \mid a_{n}-w$ i.e., $w=a_{n}+p^{k} x$ for some $x \in A_{\alpha+1}$. Now we can write $A_{\alpha+1} / A_{\alpha}=C_{1} / A_{\alpha} \oplus C_{2} / A_{\alpha}$ where $C_{1} / A_{2} \cong Q^{(p)}$ or $C_{1} / A_{2}=0$ (if $\alpha \notin E$ ) and $C_{2} / A_{\alpha} \cong \mathbf{Z}^{(\omega)}$. Then we can assume $x \in C_{2}$, since if $x=c_{1}+c_{2}$ where $c_{i} \in C_{i}$, then also $p^{k+d} \mid\left(y_{\delta}-\left(a_{n}+p^{k} c_{2}\right)\right)$ because $p^{k+d} \mid p^{k} c_{1} \bmod A_{\alpha}$. (Here we use the fact that $Q^{(p)}$ is of idempotent type.) By replacing $x$ by $x+p^{d} u$ for some $u \in C_{2}$ which is of height 1 and is independent from $x \bmod A_{\alpha}$, we can assume that $x$ is of height $1 \bmod A_{\alpha}$. (Notice that $p \nmid x+p^{d} u \bmod A_{\alpha}$ because otherwise $p \mid x \bmod A_{\alpha}$ and hence $p^{k+1} \mid y_{\delta}-a_{n} \bmod A_{\alpha}$, which contradicts the definition of $k=k(n)$.) Thus $x+A_{\alpha}$ generates a free direct summand of $A_{\alpha+1} / A_{\alpha}$ (because $C_{2} / A_{\alpha}$ is separable). Similarly, we can find $w^{\prime}=a_{n}^{\prime}+p^{k} x^{\prime}$ such that $p^{k+d} \mid y^{\prime}-w^{\prime}$ and $x^{\prime}+A_{\alpha}$ generates a free direct summand of $A_{\alpha+1}^{\prime} / A_{\alpha}^{\prime}$. By induction (use (*) with $\mu=\alpha_{n}, \nu=\alpha$ ) we can extend $g_{n}$ to $g_{n+1}: A_{\alpha+1} \rightarrow A_{\alpha+1}^{\prime}$ such that $g_{n+1}(x)=x^{\prime}$. But then $g_{n+1}(w)=$ $g_{n+1}\left(a_{n}+p^{k} x\right)=a_{n}^{\prime}+p^{k} x^{\prime}=w^{\prime}$. Thus we can let $a_{n+1}=w$.

1.5. REMARK. With appropriate modifications, Theorem 1.4 holds for groups of type $H$, where $H$ is a rational group of idempotent type, i.e., its characteristic consists of only 0's and $\infty$ 's. We shall see in the next section (Corollary 2.11) that the analog of Theorem 1.4 may fail to hold for other $H$ 's, e.g., $H=R=$ the group of rationals with square-free denominator.

2. Direct sum decompositions. Throughout this section, we shall consider a fixed $\omega_{1}$-filtration of a strongly $\omega_{1}$-free group $A=\cup_{\nu<\omega_{1}} A_{\nu}$. Let $E=\left\{\delta<\omega_{1}: A_{\delta}\right.$ is not $\omega_{1}$-pure in $\left.A\right\}$. For each $\delta \in E$ fix a sequence $\bar{y}_{\delta}=\left\{y_{\delta, i}: i<k_{\delta}\right\}$ of elements of $A_{\delta+1}$ $\left(k_{\delta} \leqslant \omega\right)$ which are linearly independent $\bmod A_{\delta}$ and satisfy

$$
A_{\delta+1} / A_{\delta}=\left\langle\bar{y}_{\delta} \cup A_{\delta}\right\rangle_{*} / A_{\delta} \oplus F_{\delta} / A_{\delta}
$$

where $F_{\delta} / A_{\delta}$ is a free group of countably infinite rank; since $\delta \in E,\left\langle\bar{y}_{\delta} \cup A_{\delta}\right\rangle_{*} / A_{\delta}$ is not free.

(It may be helpful to first read the following proofs thinking of the special case when $A$ is of type $\mathbf{Q}^{(p)}$ (see Definition 1.3) - in which case we can take $k_{\delta}=1$ and $\left\langle\bar{y}_{\delta} \cup A_{\delta}\right\rangle_{*} / A_{\delta} \cong Q^{(p)}$.)

A term in $\bar{y}_{\delta}$ is a finite linear combination of the $y_{\delta, i}$ with integer coefficients. Obviously there is a countable set of terms $t_{i}\left(\bar{y}_{\delta}\right)$, positive integers $d_{i}$, and elements $a_{i} \in A_{\delta}$ such that

$$
\left\langle\bar{y}_{\delta} \cup A_{\delta}\right\rangle_{*}=\left\langle\left\{\frac{t_{i}\left(\bar{y}_{\delta}\right)-a_{i}}{d_{i}}: i \in \omega\right\} \cup A_{\delta}\right\rangle .
$$

Our first goal is to define a ladder on $\delta$-analogous to that defined before Theorem 1.4 in the special case-whose range will give the places where new generators of $\left\langle\bar{y}_{\delta} \cup A_{\delta}\right\rangle_{*}$ first appear. We begin with the case when $k_{\delta}$ is finite. 
2.1. LemMA. Let $\delta \in E$ such that $k_{\delta}$ is finite. Then (1) the set

$$
S_{\delta} \stackrel{\text { def }}{=}\left\{\nu<\delta:\left\langle\bar{y}_{\delta} \cup A_{\nu+1}\right\rangle_{*} \neq\left\langle\bar{y}_{\delta} \cup A_{\nu}\right\rangle_{*}+A_{\nu+1}\right\}
$$

is an $\omega$-sequence whose limit is $\delta$ (i.e., $S_{\delta}$ is the range of a ladder on $\delta$ ). Moreover,

(2) if $\bar{y}_{\delta}^{\prime}=\left\{y_{\delta, i}^{\prime}: i<k_{\delta}\right\}$ is another sequence of elements of $A_{\delta+1}$ (linearly independent $\left.\bmod A_{\delta}\right)$ such that $\left\langle\bar{y}_{\delta} \cup A_{\delta}\right\rangle_{*}=\left\langle\bar{y}_{\delta}^{\prime} \cup A_{\delta}\right\rangle_{*}$, then the $\omega$-sequence

$$
S_{\delta}^{\prime}=\left\{\nu<\delta:\left\langle\bar{y}_{\delta}^{\prime} \cup A_{\nu+1}\right\rangle_{*} \neq\left\langle\bar{y}_{\delta}^{\prime} \cup A_{\nu}\right\rangle_{*}+A_{\nu+1}\right\}
$$

agrees with $S_{\delta}$ except possibly for a finite number of places.

Proof. (1) Note that $\left\langle\bar{y}_{\delta} \cup A_{\delta}\right\rangle_{*} / A_{\delta}=\cup_{\nu<\delta} H_{\nu}$ where, for all $\nu<\delta$,

$$
H_{\nu}=\left\langle\bar{y}_{\delta} \cup A_{\nu}\right\rangle_{*}+A_{\delta} / A_{\delta} \cong\left\langle\bar{y}_{\delta} \cup A_{\nu}\right\rangle_{*} / A_{\nu}
$$

is a free group of finite rank, and $H_{\nu} \neq H_{\nu+1}$ iff $\nu \in S_{\delta}$. Since $\left\langle\bar{y}_{\delta} \cup A_{\delta}\right\rangle_{*} / A_{\delta}$ is of finite rank but not finitely generated, $S_{\delta}$ must be infinite. If there exists $\mu<\delta$ such that $\left\{\nu \in S_{\delta}: \nu<\mu\right\}$ is infinite then $\left\langle\bar{y}_{\delta} \cup A_{\mu+1}\right\rangle_{*} / A_{\mu+1}$ is not finitely generated, which is impossible since $A_{\delta+1} / A_{\mu+1}$ is free.

(2) Given $\bar{y}_{\delta}^{\prime}$ as in the hypothesis, since $\bar{y}_{\delta} \subseteq\left\langle\bar{y}_{\delta}^{\prime} \cup A_{\delta}\right\rangle_{*}$ and $\bar{y}_{\delta}^{\prime} \subseteq\left\langle\bar{y}_{\delta} \cup A_{\delta}\right\rangle_{*}$, there exists $\mu<\delta$ such that $\left\langle\bar{y}_{\delta} \cup A_{\mu+1}\right\rangle_{*}=\left\langle\bar{y}_{\delta}^{\prime} \cup A_{\mu+1}\right\rangle_{*}$. But then, for all $\left.\nu\right\rangle$ $\mu+1$,

$$
\left\langle\bar{y}_{\delta} \cup A_{\nu+1}\right\rangle_{*}=\left\langle\left\langle\bar{y}_{\delta} \cup A_{\mu+1}\right\rangle_{*} \cup A_{\nu+1}\right\rangle_{*}=\left\langle\bar{y}_{\delta}^{\prime} \cup A_{\nu+1}\right\rangle_{*},
$$

so $\nu \in S_{\delta}$ iff $\nu \in S_{\delta}^{\prime}$.

In the case when $k_{\delta}$ is infinite $S_{\delta}$ will not be an $\omega$-sequence unless the $y_{\delta, i}$ are chosen with some care.

2.2. Lemma. Let $\delta \in E$ such that $k_{\delta}=\omega$.

(1) Suppose there is a ladder $\sigma: \omega \rightarrow \delta$ on $\delta$ such that, for all $n \in \omega$,

$$
\left\langle\bar{y}_{\delta} \cup A_{\sigma(n)+1}\right\rangle_{*}=\left\langle\left\{y_{\delta, j}: j \leqslant n-1\right\} \cup A_{\sigma(n)+1}\right\rangle_{*}+\left\langle\bar{y}_{\delta}\right\rangle .
$$

Then $S_{\delta}$ (defined as in (2.1.1)) is an w-sequence. Moreover, if $\left\{y_{\delta, 0}, \ldots, y_{\delta, m}\right\}$ is pure-independent $\bmod A_{\sigma(m+1)+1}$ then the least element of $S_{\delta}$ is $>\sigma(m+1)$.

(2) For any ladder $\sigma: \omega \rightarrow \delta$ on $\delta$ there is a sequence $\bar{y}_{\delta}=\left\{y_{\delta, i}: i<\omega\right\}$ of elements of $A_{\delta+1}$ which are linearly independent $\bmod A_{\delta}$ and satisfy (2.2.1) such that

$$
A_{\delta+1} / A_{\delta}=\left\langle\bar{y}_{\delta} \cup A_{\delta}\right\rangle_{*} / A_{\delta} \oplus F_{\delta} / A_{\delta}
$$

where $F_{\delta} / A_{\delta} \cong \mathbf{Z}^{(\omega)}$.

Proof. (1) If $S_{\delta}^{n}$ is defined as in (2.1.1) using $\bar{y}_{\delta}^{n} \stackrel{\text { def }}{=}\left\{y_{\delta, 0}, \ldots, y_{\delta, n}\right\}$ instead of $\bar{y}_{\delta}$, then, using (2.2.1) and the linear independence of $\bar{y}_{\delta}$ over $A_{\delta}$, one can prove that

$$
S_{\delta} \cap[0, \sigma(n)]=S_{\delta}^{n-1} \cap[0, \sigma(n)] .
$$

Hence, since each $S_{\delta}^{n}$ is an $\omega$-sequence by $2.1, S_{\delta}=\cup_{n} S_{\delta}^{n}$ is an $\omega$-sequence. Moreover, since

$$
S_{\delta} \cap[0, \sigma(m+1)]=S_{\delta}^{m} \cap[0, \sigma(m+1)],
$$

if $\left\{y_{\delta, 0}, \ldots, y_{\delta, m}\right\}$ is pure independent $\bmod A_{\sigma(m+1)+1}$, then $S_{\delta} \cap[0, \sigma(m+1)]=\varnothing$. 
(2) Let $\bar{y}_{\delta}$ be as in the introduction to this section (so in particular, $\bar{y}_{\delta}$ is linearly independent $\bmod A_{\delta}$ and (2.2.2) holds). We shall define by induction on $n$ a new sequence $\bar{y}_{\delta}^{\prime}$ such that (2.2.1) holds for this sequence and, for all $i<\omega, y_{\delta, i}+A_{\sigma(n)+2}$ $=y_{\delta, i}^{\prime}+A_{\sigma(n)+2}$. Suppose that $y_{\delta, i}^{\prime}$ has been defined for all $i \leqslant n-1$. Let $y_{\delta, n}^{\prime}=y_{\delta, n}$ $+u_{n}$, where $u_{n} \in A_{\sigma(n)+2}$ is of height $1 \bmod A_{\sigma(n)+1}$ and is independent $\bmod A_{\sigma(n)+1}$ from the first components of $y_{\delta, 0}^{\prime}, \ldots, y_{\delta, n-1}^{\prime}, y_{\delta, n}$ in a decomposition

$$
A_{\delta+1} / A_{\sigma(n)+1}=\left(A_{\sigma(n)+2} / A_{\sigma(n)+1}\right) \oplus D
$$

(where $\left.D \cong A_{\delta+1} / A_{\sigma(n)+2}\right)$. Now notice that by construction if $z=\sum_{i=0}^{n} r_{i} y_{\delta, i}^{\prime}\left(r_{i} \in\right.$ Z) and if $q$ divides $z \bmod A_{\sigma(n)+1}$ then $q$ divides $r_{n}$. This is sufficient to imply (2.2.1).

2.3. Definition. Let $A=\cup_{\nu<\omega_{1}} A_{\nu}, E$ and $\bar{y}_{\delta}$ be as in the introduction to this section, and moreover, let $\bar{y}_{\delta} \subseteq A_{\delta+1}(\delta \in E)$ be chosen so that (2.2.1) holds for some ladder $\sigma$ on $\delta$. Given the $\omega_{1}$-filtration and the $\bar{y}_{\delta}$ there is for each $\delta \in E$ a unique ladder $\eta_{\delta}$ on $\delta$ whose range is $S_{\delta}$ (cf. (2.1.1)) -called the associated ladder to $A$ at $\delta$; the set $\left\{\eta_{\delta}: \eta \in E\right\}$ is called the associated ladder system to $A$. (It is, of course, not an invariant of $A$ : it depends upon the choice of $\omega_{1}$-filtration and of the $\bar{y}_{\delta}$.)

The following will be our main tool in constructing direct sum decompositions of $A$; it is a theorem of ZFC.

2.4. Theorem. Let $A=\cup_{\nu<\omega_{1}} A_{\nu}, \bar{y}_{\delta}$ and $\left\{\eta_{\delta}: \delta \in E\right\}$ be as in Definition 2.3. Suppose there is a partition $E=\amalg_{\beta<\omega_{1}} E^{\beta}$ such that, for all $\beta$ and all $\delta \in E^{\beta}, \delta>\beta$, and for all sufficiently large $n, \eta_{\delta}(n) \notin E-E^{\beta}$.

Suppose also that for each $\beta<\omega_{1}$ there is a pure subgroup $B^{\beta}$ of $A$ such that for all $\beta<\omega_{1}$ and all $\nu<\omega_{1}$, if $B_{\nu}^{\beta} \stackrel{\text { def }}{=} B^{\beta} \cap A$

(0) for all $\delta \in E^{\beta}, \bar{y}_{\delta} \subseteq B_{\delta+1}^{\beta}$;

(i) $E^{\beta}=\left\{\nu<\omega_{1}: B_{\nu}^{\beta}\right.$ is not $\omega_{1}$-pure in $\left.B^{\beta}\right\}$;

(ii) if $\nu>\beta, B_{\nu+1}^{\beta} / B_{\nu}^{\beta} \cong B_{\nu+1}^{\beta} / B_{\nu}^{\beta} \oplus \mathbf{Z}^{(\omega)}$; and

(iii) if $\nu>\beta, B_{\nu+1}^{\beta}+A_{\nu}$ is pure in $A_{\nu+1}$.

Then $A$ is filtration-equivalent to $\bigoplus_{\beta<\omega_{1}} B^{\beta}$.

Proof. Without loss of generality, redefine $B_{\nu}^{\beta}=0$ for $\nu \leqslant \beta$. Then $B_{\nu}=\bigoplus_{\beta<\omega_{1}} B_{\nu}^{\beta}$ defines an $\omega_{1}$-filtration of $\bigoplus_{\beta<\omega_{1}} B^{\beta}$. It suffices to prove

for all $\mu<\nu<\omega_{1}$, given a 1.p. isomorphism $f: A_{\mu+1} \rightarrow B_{\mu+1}$ and given $x_{0}, \ldots, x_{m} \in B_{\nu+1}^{\gamma}$ which are pure independent $\bmod A_{\nu}$, where $\nu \notin E-E^{\gamma}$, there is a 1.p. isomorphism $\tilde{f}$ : $A_{\nu+1} \rightarrow B_{\nu+1}$ extending $f$ such that for all $j \leqslant m, \tilde{f}\left(x_{j}\right)=x_{j}$.

The proof is by induction on $\nu$; there are 3 cases, 2 of them easy.

Case 1. $\nu=\tau+1$ for some $\tau$. By induction we may assume that $\tau=\mu$. Then since $A_{\nu+1} / A_{\mu+1}$ (resp. $B_{\nu+1} / B_{\mu+1}$ ) is free, $x_{0}+A_{\mu+1}, \ldots, x_{m}+A_{\mu+1}$ are a basis of a summand of $A_{\nu+1} / A_{\mu+1}$ and $B_{\nu+1} / B_{\mu+1}$, and it is clear that we can extend $f$ : $A_{\mu+1} \rightarrow B_{\mu+1}$ to $\tilde{f}: A_{\nu+1} \rightarrow B_{\nu+1}$ so that $\tilde{f}\left(x_{j}\right)=x_{j}$. 
Case 2. $\nu \in \operatorname{Lim}\left(\omega_{1}\right)-E$. Choose a strictly increasing sequence $\alpha_{n}$ approaching $\nu$ such that $\alpha_{0}>\mu$. Then by induction define a chain of 1.p. isomorphisms $g_{n}$ : $A_{\alpha_{n}+1} \rightarrow B_{\alpha_{n}+1}$ extending $f$. If $g \stackrel{\text { def }}{=} \cup_{n \in \omega} g_{n}$ then $g$ is a 1.p. isomorphism: $A_{\nu} \rightarrow B_{\nu}$ and since $A_{\nu+1} / A_{\nu}$ and $B_{\nu+1} / B_{\nu}$ are free, we can extend to $\tilde{f}$ just as in Case 1 .

Case 3. $\nu \in E$. Say $\nu=\delta \in E^{\gamma}$. Suppose we are given $\mu, f$ and $x_{0}, \ldots, x_{m}$ as in (*). Since $\delta$ is fixed, let us write $\bar{y}$ instead of $\bar{y}_{\delta}$, and $y_{j}$ instead of $y_{\delta, j}$. Because changing the sequence $\bar{y}$ in finitely many places will only change finitely many values of $\eta_{\delta}$ (cf. Lemma 2.1(2)) and because $x_{0}, \ldots, x_{m}$ belong to $B_{\delta+1}^{\gamma}$ and are linearly independent $\bmod A_{\delta}$, we can assume without loss of generality that $y_{j}=x_{j}$ for $j \leqslant m$. Moreover, we can assume that $\bar{y}$ satisfies (2.2.1) with $\sigma(m+1) \geqslant \mu$, so, since $x_{0}, \ldots, x_{m}$ are pure-independent $\bmod A_{\delta}$, by $2.2(1), \eta_{\delta}(0)>\mu$.

By hypothesis there is an $N$ such that for $n \geqslant N, \eta_{\delta}(n) \notin E-E^{\gamma}$; let $r(>m+1)$ be such that $\sigma(r) \geqslant \eta_{\delta}(N)$. By replacing $y_{j}$ by $y_{j}+u_{j}$ for appropriate $u_{j}$ 's in $B_{\sigma(r)+2}^{\gamma}$, $j=m+1, \ldots, r-1$ (cf. proof of 2.2), we can get $S_{\delta} \cap[0, \sigma(r)]=S_{\delta}^{m} \cap[0, \sigma(r)]$, without changing $S_{\delta} \cap[\sigma(r)+2, \delta\rangle$. Then, since $x_{0}, \ldots, x_{m}$ are pure-independent $\bmod A_{\delta}\left(\right.$ and hence $\left.\bmod A_{\sigma(r)+1}\right), S_{\delta} \cap[0, \sigma(r)]=\varnothing$, so for all $n \in \omega, \eta_{\delta}(n) \notin E$ - $E^{\gamma}$.

Notice also by (0) and (iii), $\left\langle\bar{y} \cup B_{\delta}^{\gamma}\right\rangle_{*}+A_{\delta}=\left\langle\bar{y} \cup A_{\delta}\right\rangle_{*}$; so

$$
A_{\delta+1} / A_{\delta}=\left(\left\langle\bar{y} \cup B_{\delta}^{\gamma}\right\rangle_{*}+A_{\delta}\right) / A_{\delta} \oplus F_{\delta} / A_{\delta}
$$

and by (i),

$$
B_{\delta+1} / B_{\delta}=\left\langle\bar{y} \cup B_{\delta}^{\gamma}\right\rangle_{*} / B_{\delta}^{\gamma} \oplus F_{\delta}^{\prime} / B_{\delta}
$$

where $F_{\delta} / A_{\delta} \cong \mathbf{Z}^{(\omega)} \cong F_{\delta}^{\prime} / B_{\delta}$.

Finally, notice that $\eta_{\delta}$ is the associated ladder to $B^{\gamma}$ at $\delta$ (determined by $\bar{y}$ ). For this, we show that, for all $\mu<\delta,\left\langle\bar{y} \cup B_{\mu+1}^{\gamma}\right\rangle_{*} \neq\left\langle\bar{y} \cup B_{\mu}^{\gamma}\right\rangle_{*}+B_{\mu+1}^{\gamma}$ iff $\left\langle\bar{y} \cup A_{\mu+1}\right\rangle_{*} \neq\left\langle\bar{y} \cup A_{\mu}\right\rangle_{*}+A_{\mu+1}$. It is not hard to see that this will follow if we show that for all $\nu<\delta$, all terms $t=t(\bar{y})$ and all $d \in \omega-\{0\}$, if $d \mid t \bmod A_{\nu}$ then $d \mid t \bmod B_{\nu}^{\gamma}$. If false, then there is a $\nu<\delta, a \in A_{\nu}, b \in B_{\nu+1}^{\gamma}$ such that $d \mid t-a$ and $d \mid t-b$ but $d \nmid t \bmod B_{\nu}^{\gamma}$; thus $d \mid a-b$, or $d \mid b \bmod A_{\nu}$. Now (iii) implies $B_{\nu+1}^{\gamma} / B_{\nu}^{\gamma}$ is a pure subgroup of $A_{\nu+1} / A_{\nu}$, so $d \mid b \bmod B_{\nu}^{\gamma}$. Hence, $d \mid t \bmod B_{\nu}^{\gamma}$, a contradiction.

After all this preparation we can begin the construction of $\tilde{f}: A_{\delta+1} \rightarrow B_{\delta+1}$ extending $f$. For all $n$, let $\alpha_{n}=\eta_{\delta}(n)$. We shall define by induction on $n$ a chain of level-preserving isomorphisms

$$
g_{n}: A_{\alpha_{n}+1} \rightarrow B_{\alpha_{n}+1}
$$

Simultaneously we shall define finitely many terms $t_{l}^{n}(\bar{y})$, positive integers $d_{l}^{n}$ and elements $a_{l}^{n}$ in $B_{\alpha_{n}+1}^{\gamma}\left(l \leqslant r_{n}\right)$ such that for all $l \leqslant r_{n}, g_{n}\left(a_{l}^{n}\right)=a_{l}^{n}$ and $\left\langle\bar{y} \cup B_{\alpha_{n}+1}^{\gamma}\right\rangle_{*}$ $=\left\langle\bar{y} \cup B_{\alpha_{n}}^{\gamma}\right\rangle_{*}+\left\langle Z_{n}\right\rangle$ where

$$
Z_{n}=\left\{\frac{t_{l}^{n}(\bar{y})-a_{l}^{n}}{d_{l}^{n}}: l \leqslant r_{n}\right\} .
$$


Suppose for the moment that we can do this; then

$$
\left\langle\bar{y} \cup B_{\delta}^{\gamma}\right\rangle_{*}=\left\langle\bigcup_{n} Z_{n} \cup B_{\delta}^{\gamma}\right\rangle .
$$

Thus we can extend $g \stackrel{\text { def }}{=} \cup_{n} g_{n}: A_{\delta} \rightarrow B_{\delta}$ to a l.p. isomorphism $\tilde{f}: A_{\delta+1} \rightarrow B_{\delta+1}$ by sending $\left(d_{l}^{n}\right)^{-1}\left(t_{l}^{n}(\bar{y})-a_{l}^{n}\right)$ to itself and sending a basis of $F_{\delta} \bmod A_{\delta}$ onto a basis of $F_{\delta}^{\prime} \bmod B_{\delta}$ (cf. (2.4.1) and (2.4.2)).

Thus it remains to define the $g_{n}, a_{l}^{n}$ etc. Suppose this has been done for $g_{n-1}$ (where $g_{-1}=f, \alpha_{-1}=\mu$ and $r_{-1}=-1$; notice that $\left\langle\bar{y} \cup B_{\mu+1}\right\rangle_{*}=\left\langle\bar{y} \cup B_{\mu+1}\right\rangle$ ).

Let

$$
H_{n}=\left\langle\bar{y} \cup B_{\alpha_{n}}^{\gamma}\right\rangle_{*}+B_{\alpha_{n}+1}^{\gamma} \text { and } G_{n}=\left\langle\bar{y} \cup B_{\alpha_{n}+1}^{\gamma}\right\rangle_{*},
$$

so $G_{n} / H_{n}$ is a torsion-group, which is finitely-generated because of (2.2.1). Thus by the Fundamental Theorem there are finitely many elements

$$
z_{l}^{n}=\frac{\left(t_{l}^{n}(\bar{y})-a_{l}^{n}\right)}{d_{l}^{n}}
$$

$\left(l \leqslant r_{n}\right)$ of $G_{n}$ such that

$$
G_{n} / H_{n}=\bigoplus_{l=0}^{r_{n}}\left\langle z_{l}^{n}+H_{n}\right\rangle
$$

and each $z_{l}^{n}+H_{n}$ has order $p_{l}^{m_{l}}$ for some prime $p_{l}$ and some $m_{l} \geqslant 1$. (Since $n$ is fixed, we shall omit the index $n$ on $p_{l}$ and $m_{l}$, and also from now on, write $z_{l}$ for $z_{l}^{n}, d_{l}$ for $d_{l}^{n}$, and $r$ for $r_{n}$.)

A crucial observation is that, since $\eta_{\delta}$ is the associated ladder to $B^{\gamma}$ at $\delta$,

$$
\left\langle\bar{y} \cup B_{\alpha_{n}}^{\gamma}\right\rangle_{*}=\left\langle\bar{y} \cup B_{\alpha_{n-1}+1}^{\gamma}\right\rangle_{*}+B_{\alpha_{n}}^{\gamma},
$$

so

$$
H_{n}=\left\langle\bar{y} \cup B_{\alpha_{n-1}+1}^{\gamma}\right\rangle_{*}+B_{\alpha_{n}+1}^{\gamma} \text {. }
$$

Now, since $d_{l} z_{l} \in H_{n}, p_{l}^{m_{l}}$ divides $d_{l}$; let $e_{l}$ be the quotient. Then $e_{l}^{-1}\left(t_{l}(\bar{y})-a_{l}\right) \in$ $H_{n}$, so by (2.4.3) and by induction there exists $b_{l} \in B_{\alpha_{n-1}+1}^{\gamma}$ such that $e_{l}$ divides $a_{l}-b_{l}$ and $g_{n-1}\left(b_{l}\right)=b_{l}$. Thus, $a_{l}=b_{l}+e_{l} x_{l}$ for some $x_{l} \in B_{\alpha_{n}+1}^{\gamma}$. By replacing $x_{l}$ by $x_{l}+p_{l}^{m_{l}} u_{l}(l=0, \ldots, r)$, where the $u_{l} \in B_{\alpha_{n}+1}^{\gamma}$ are pure-independent $\bmod \left\langle B_{\alpha_{n}}^{\gamma}, x_{0}, \ldots, x_{r}\right\rangle$, we can assume that the $x_{l}$ 's are independent and that if $q$ is a prime

$$
q \mid \sum_{l=0}^{r} k_{l} x_{l} \bmod B_{\alpha_{n}}^{\gamma} \quad \text { implies } q=p_{l} \text { for all } l \text { s.t. } q \nmid k_{l} .
$$

We claim that $x_{0}, \ldots, x_{r}$ are pure-independent $\bmod A_{\alpha_{n}}$. If true, we are done, for we can apply (*) with $\mu=\alpha_{n-1}, \nu=\alpha_{n}, f=g_{n-1}$ and let $g_{n}=\tilde{f}$. (Notice that here we need that $\alpha_{n} \in E^{\gamma}$ if $\alpha_{n} \in E$.)

Thus it remains to prove the claim. Since $A_{\alpha_{n}+1} /\left(B_{\alpha_{n}+1}^{\gamma}+A_{\alpha_{n}}\right)$ is torsion-free, it is enough to prove that $\left\{x_{0}, \ldots, x_{r}\right\} \subseteq B_{\alpha_{n}+1}^{\gamma}$ is pure-independent $\bmod B_{\alpha_{n}}^{\gamma}$. So suppose $q$ is a prime such that $q \mid \sum_{l=0}^{s} k_{l} x_{l} \bmod B_{\alpha_{n}}^{\gamma}$, for some $s \leqslant r$; i.e., $\sum_{l=0}^{s} k_{l} x_{l}=q w+z$, 
where $w \in A_{\alpha_{n}+1}, z \in B_{\alpha_{n}}^{\gamma}$. By (2.4.4) and renumbering we may assume that $q=p_{l}$ for all $l \leqslant s$. We shall show that

$$
\sum_{l=0}^{s} q^{m_{l}-1} k_{l} z_{l}
$$

belongs to $H_{n}$, which implies that $q \mid k_{l}$ for all $l \leqslant s$. because $z_{l}+H_{n}$ has order $q^{m_{l}}$ $\left(=p_{l}^{m_{l}}\right)$. Now $(2.4 .5)=$

$$
\sum_{l=0}^{s}\left(q e_{l}\right)^{-1} k_{l}\left(t_{l}(\bar{y})-\left(b_{l}+e_{l} x_{l}\right)\right)=(q e)^{-1}\left(\xi-e \sum_{l=0}^{s} k_{l} x_{l}\right)
$$

where $e=e_{0} e_{1} \cdots e_{s}$ and

$$
\xi \in\left\langle\bar{y} \cup B_{\alpha_{n-1}+1}^{\gamma}\right\rangle=(q e)^{-1}(\xi-e(q w+z))=\left((q e)^{-1}(\xi-z)\right)-w
$$

which clearly belongs to $H_{n}$ since $z \in B_{\alpha_{n}}^{\gamma}$.

2.5. Corollary. If $B$ is a subgroup of a strongly $\omega_{1}$-free group $A$ of cardinality $\omega_{1}$ such that $A / B \cong C \oplus F$ where $C$ is countable and $F$ is free, then $B$ is filtration-equivalent to $A$. Hence, assuming $M A+\neg C H, B$ is isomorphic to $A$.

Proof. Let us write $A / B=H_{0} / B \oplus H_{1} / B$, where $H_{0} / B \cong C$ and $H_{1} / B$ is free. Then we can choose an $\omega_{1}$-filtration $A=\cup_{\nu<\omega_{1}} A_{\nu}$ such that for all $\nu<\omega_{1}$,

$$
\left(A_{\nu}+B\right) / B=H_{0} / B \oplus H_{1, \nu} / B
$$

where $H_{1} / H_{1, \nu}$ is free. Thus for all $\nu<\omega_{1}, A /\left(B+A_{\nu}\right)$ is free; so

$$
A / A_{\nu} \cong\left(B+A_{\nu}\right) / A_{\nu} \oplus A /\left(B+A_{\nu}\right)
$$

and $A /\left(B+A_{\nu}\right)$ is free. Hence, for all $\nu, A_{\nu+1} /\left(B_{\nu+1}+A_{\nu}\right)$ is free. By choosing a subsequence if necessary, we can also assume that for all $\nu<\omega_{1}$, if $B_{\nu}=B \cap A_{\nu}$, $B_{v+1} / B_{\nu} \cong B_{v+1} / B_{\nu} \oplus \mathbf{Z}^{(\omega)}$. Hence, if we let $E=\left\{\delta<\omega_{1}: A_{\delta}\right.$ is not $\omega_{1}$-pure in $\left.A\right\}$, and for $\delta \in E$ choose $\bar{y}_{\delta} \subseteq B$ independent over $B_{\delta}$ such that $B_{\delta+1}=\left\langle\bar{y}_{\delta} \cup B_{\delta}\right\rangle_{*}$, then we can apply Theorem 2.4 with $E^{0}=E, B^{0}=B+A_{0}$ and for $\beta>0, E^{\beta}=\varnothing$, $B^{\beta}=0$. Therefore $A$ is filtration-equivalent to $B+A_{0}$, which is filtration-equivalent to $B$.

Corollary 2.5 fails in a model of $\mathrm{CH}$ (see Theorem 3.7).

2.6. Corollary. If $A$ is a strongly $\omega_{1}$-free group of cardinality $\omega_{1}$ then $A$ is filtration-equivalent to $A \oplus \mathbf{Z}^{\left(\omega_{1}\right)}$. Hence, assuming $M A+\neg C H, A \cong A \oplus \mathbf{Z}^{\left(\omega_{1}\right)}$.

Proof. Apply 2.5 with $A=A \oplus \mathbf{Z}^{\left(\omega_{1}\right)}$ and $B=A$.

The next theorem will imply the existence of the subgroups $B^{\beta}$ satisfying (0)-(iii) of Theorem 2.4.

2.7. ThEOREM. Let $A$ be a strongly $\omega_{1}$-free group of cardinality $\omega_{1}$, and let $\left\{A_{\nu}\right.$ : $\left.\nu<\omega_{1}\right\}, E$ and $\bar{y}_{\delta}(\delta \in E)$ be as in Definition 2.3. Then for any $E^{\prime} \subseteq E$ there is a pure subgroup $B$ of $A$ such that, if we define $B_{\nu}=B \cap A_{\nu}$, we have

(0) for all $\delta \in E^{\prime}, \bar{y}_{\delta} \subseteq B_{\delta+1}$,

(i) $E^{\prime}=\left\{\nu<\omega_{1}: B_{\nu}\right.$ is not $\omega_{1}$-pure in $\left.A\right\}$,

(ii) for all $\nu<\omega_{1}, B_{\nu+1} / B_{\nu} \cong B_{\nu+1} / B_{\nu} \oplus \mathbf{Z}^{(\omega)}$, and

(iii) for all $\nu<\omega_{1}, B_{\nu+1}+A_{\nu}$ is pure in $A_{\nu+1}$. 
Proof. We shall define by induction on $\nu<\omega_{1}$ a continuous chain of pure subgroups $B_{\nu}$ of $A_{\nu}$ such that

(a) for all $\mu<\nu, B_{\nu} \cap A_{\mu}=B_{\mu}$,

(b) for all $\nu<\omega_{1}, B_{\nu+1} / B_{\nu} \cong B_{\nu+1} / B_{\nu} \oplus \mathbf{Z}^{(\omega)}$,

(c) if $\nu \notin E-E^{\prime}, A_{\nu+1}=B_{\nu+1}+A_{\nu}$, and

(d) for all $\nu<\omega_{1}, a \in A_{\nu}$, and $d \in \omega-\{0\}$, there exists $b \in B_{\nu}$ such that $d \mid(b-a)$.

Let $A_{-1}=0=B_{-1}$ and suppose $\nu \in \omega_{1}$ such that $B_{\mu}$ has been defined for all $\mu<\nu$. If $\nu \in \operatorname{Lim}\left(\omega_{1}\right)$, let $B_{\nu}=\cup_{\mu<\nu} B_{\mu}$. Otherwise $\nu=\delta+1$ for some $\delta$; then we can write

$$
A_{\delta+1} / A_{\delta}=\left\langle\bar{y}_{\delta} \cup A_{\delta}\right\rangle_{*} / A_{\delta} \oplus \bigoplus_{m=1}^{\infty} F_{m} / A_{\delta}
$$

where for all $m \in \omega, F_{m} / A_{\delta} \cong \mathbf{Z}^{(\omega)}$, and $\bar{y}_{\delta}(=\varnothing$ if $\delta \notin E)$ is a sequence of elements linearly independent over $A_{\delta}$. Let $\left\{a_{n}^{m}+A_{\delta}: n \in \omega\right\}$ be a basis of $F_{m} / A_{\delta}$. Let $F_{0}=\left\langle\bar{y}_{\delta} \cup A_{\delta}\right\rangle_{*}$ (so $F_{0}=A_{\delta}$ if $\delta \notin E$ ). For each $m \in \omega$, let $\left\{\left(x_{n}^{m}, d_{n}^{m}\right): n \in \omega\right\}$ be an enumeration of $\left\langle\cup_{k \leqslant m} F_{k}\right\rangle \times(\omega-\{0\})$. Then for each $m \in \omega$ let $b_{n}^{m}=x_{n}^{m}+$ $d_{n}^{m} a_{n}^{m+1}$, and let $\bar{b}^{m}=\left\{b_{n}^{m}: n \in \omega\right\}$. Let $\bar{b}=\cup_{m \in \omega} \bar{b}^{m}$. By induction on $m$ one can prove that $\bar{y}_{\delta} \cup \bar{b}^{0} \cup \cdots \cup \bar{b}^{m}$ is linearly independent over $A_{\delta}$; also, by Pontryagin's criterion, $\left\langle B_{\delta} \cup \bar{b}\right\rangle_{*} / B_{\delta}$ is free. If $\delta \notin E-E^{\prime}$, let $B_{\delta+1}=\left\langle B_{\delta} \cup \bar{y}_{\delta} \cup \bar{b}\right\rangle_{*}$. If $\delta \in E-E^{\prime}$, let $B_{\delta+1}=\left\langle B_{\delta} \cup \bar{b}\right\rangle_{*}$. By construction (d) holds; let us verify (c). Suppose $\delta \notin E-E^{\prime}$. If $a \in A_{\delta+1}$ then by construction there exists $t \in\left\langle\bar{y}_{\delta} \cup \bar{b}\right\rangle$, $u \in A_{\delta}$, and $d \in \omega-\{0\}$ such that $d a=t-u$. By (d) there exists $b \in B_{\delta}$ such that $d \mid(b-u)$. But then $d$ divides $t-b$ so $d^{-1}(t-b) \in B_{\delta+1}$ and, hence,

$$
a=d^{-1}(t-b)+d^{-1}(b-u) \in B_{\delta+1}+A_{\delta} .
$$

Let us verify (a). Suppose $\delta \notin E-E^{\prime}$ and $z \in B_{\delta+1} \cap A_{\delta}$. Since $z \in B_{\delta+1}$, there exists $d \in \omega-\{0\}$ such that $d z=t-c$ where $t \in\left\langle\bar{y}_{\delta} \cup \bar{b}\right\rangle$ and $c \in B_{\delta}$. Also since $z \in A_{\delta}, t-c \in A_{\delta}$, which implies $t=0$ since $c \in A_{\delta}$, and $\bar{y} \cup \bar{b}$ is linearly independent over $A_{\delta}$. Therefore $d z=c \in B_{\delta}$, so $z \in B_{\delta}$. The proof is similar if $\delta \in E-E^{\prime}$.

Finally we must verify (b). If $\delta \notin E-E^{\prime}$ then, by (c), $B_{\delta+1} / B_{\delta} \cong A_{\delta+1} / A_{\delta}$, so (b) holds by choice of the $\omega_{1}$-filtration of $A$ (see introduction to $\S 1$ ). If $\delta \in E-E^{\prime}$ then $B_{\delta+1} / B_{\delta}=\left\langle B_{\delta} \cup \bar{b}\right\rangle_{*} / B_{\delta}$ is free and clearly not finitely-generated.

This completes the construction of the $B_{\nu}$. Let $B=\cup_{\nu<\omega_{1}} B_{\nu}$. By construction, (0) and (ii) hold; and (d) implies (iii). As for (i), $E^{\prime} \subseteq\left\{\nu<\omega_{1}: B_{\nu}\right.$ is not $\omega_{1}$-pure in $\left.B\right\}$ since for $\delta \notin E-E^{\prime}$, by (c), $B_{\delta+1} / B_{\delta} \cong A_{\delta+1} / A_{\delta}$. The opposite inclusion holds because-as noted above- $B_{\delta+1} / B_{\delta}$ is free if $\delta \in E-E^{\prime}$, and $B_{\mu} / B_{\delta+1}$ is free for all $\delta<\mu<\omega_{1}$ since, by (a), $B_{\mu} / B_{\delta+1}$ is isomorphic to a subgroup of $A_{\mu} / A_{\delta+1}$.

2.8. THEOREM (MA $+\neg \mathrm{CH})$. If $A$ is an $\omega_{1}$-separable group of cardinality $\omega_{1}$ which is not free, then $A \cong \bigoplus_{\beta<\omega_{1}} B^{\beta}$ for some nonfree groups $B^{\beta}$.

Proof. Let $A=\cup_{\nu<\omega_{1}} A_{\nu}, E, \bar{y}_{\delta}(\delta \in E)$ and $\left\{\eta_{\delta}: \delta \in E\right\}$ be as in Definition 2.3. By Theorem 0.5 there is a partition of $E$ into disjoint stationary sets, $E=\amalg_{\beta<\omega_{1}} E^{\beta}$ s.t. for all $\beta$ and all $\delta \in E^{\beta}, \delta>\beta$ and only finitely many members of the range of 
$\eta_{\delta}$ belong to $E-E^{\beta}$. For each $E^{\beta}$, let $B^{\beta}$ be a pure subgroup of $A$ constructed as in 2.7, so that in particular (if $B_{\nu}^{\beta} \stackrel{\text { def }}{=} B^{\beta} \cap A_{\nu}$ ),

$$
E^{\beta}=\left\{\nu<\omega_{1}: B^{\beta} / B_{\nu}^{\beta} \text { is not } \omega_{1} \text {-free }\right\}
$$

and for all $\delta \in E^{\beta}, \bar{y}_{\delta} \subseteq B_{\delta+1}^{\beta}$ and if $\nu \notin E-E^{\beta}, A_{\nu+1}=B_{\nu+1}^{\beta}+A_{\nu}$. Then Theorem 2.4 applies and $A$ is filtration-equivalent to $\bigoplus_{\beta<\omega_{1}} B^{\beta}$. Hence by Theorem 1.2, $A \cong \bigoplus_{\beta<\omega_{1}} B^{\beta}$.

Note that in the above proof the hypothesis $\mathrm{MA}+\neg \mathrm{CH}$ is used twice: first (by Theorem 0.5) to get the decomposition $E=\prod_{\beta<\omega_{1}} E^{\beta}$; second, in order to apply the classification theorem (Theorem 1.2). The observation that in some models of $\mathrm{MA}+\neg \mathrm{CH}$, the conclusion of Theorem 0.5 holds (modulo a cub) for any decomposition of $E$ (cf. Theorem $0.8(2)$ ) leads to the following (cf. remarks after Lemma $1.1)$.

2.9. Definition. Say that an $\omega_{1}$-separable group $A$ with $\Gamma(A)=\tilde{E}$ has the decomposition property if whenever $\tilde{E}=\bigvee\left\{\tilde{E}^{\beta}: \beta<\omega_{1}\right\}$ where $\tilde{E}^{\beta} \cap \tilde{E}^{\gamma}=\tilde{\varnothing}$ for all $\gamma \neq \beta$, we can write $A=\bigoplus_{\beta<\omega_{1}} A_{\beta}$ where for all $\beta<\omega_{1}, \Gamma\left(A_{\beta}\right)=\tilde{E}^{\beta}$.

Using Theorem 1.4 one can prove that in any model of $\mathrm{ZFC}+\mathrm{MA}+\neg \mathrm{CH}$, every $\omega_{1}$-separable group of type $Q^{(p)}$ has the decomposition property. However, for arbitrary $\omega_{1}$-separable groups the problem is undecidable in $\mathrm{ZFC}+\mathrm{MA}+\neg \mathrm{CH}$.

2.10. TheOREM. (1) There is a model of $\mathrm{ZFC}+\mathrm{MA}+{ }_{\neg} \mathrm{CH}$ in which there is an $\omega_{1}$-separable group of cardinality $\omega_{1}$ which does not have the decomposition property.

(2) There is a model of $\mathrm{ZFC}+\mathrm{MA}+{ }_{\neg} \mathrm{CH}$ in which every $\omega_{1}$-separable group of cardinality $\omega_{1}$ has the decomposition property.

Proof. (1) We shall use the model in Theorem 0.8(1). Let $E_{0}, E_{1}$ and $\left\{\eta_{\delta}: \delta \in E_{0}\right\}$ be such that $(*)$ holds. For every $\delta \in E_{1}$, let $\eta_{\delta}$ be an arbitrary ladder on $\delta$. Let $E=E_{0} \cup E_{1}$. We shall construct an $\omega_{1}$-separable group $A$ of type $R$ (cf. 1.3) such that $\Gamma(A)=\tilde{E}$ and $A$ does not have the decomposition property; in particular, $A$ is not the direct sum of groups $A_{0}$ and $A_{1}$ such that $\Gamma\left(A_{0}\right)=\tilde{E}_{0}$ and $\Gamma\left(A_{1}\right)=\tilde{E}_{1}$. (Recall that $R$ is the group of rationals with square-free denominators.) We shall define $A$ as a subgroup of

$$
D=\bigoplus_{\nu<\omega_{1}} \mathbf{Q} x_{\nu} \oplus \bigoplus_{\delta \in E} \mathbf{Q} y_{\delta} .
$$

For each $n \in \omega$ and $\delta \in E$, if $p_{n}$ denotes the $n$th prime, define $z_{\delta, n}$ by induction on $\delta$ as follows: if $\eta_{\delta}(n)=\gamma$ and $\gamma \notin E$,

$$
z_{\delta, n}=\frac{y_{\delta}-x_{\gamma}}{p_{n}},
$$

and if $\gamma \in E$,

$$
z_{\delta, n}=\frac{y_{\delta}-z_{\gamma, n}}{p_{n}} .
$$


Let $A$ be the subgroup of $D$ generated by $\left\{x_{\nu}: \nu<\omega_{1}\right\} \cup\left\{z_{\delta, n}: \delta \in E, n \in \omega\right\}$. Define an $\omega_{1}$-filtration of $A$ by

$$
A_{\mu}=A \cap\left(\bigoplus_{\nu<\mu} \mathbf{Q} x_{\nu} \oplus \bigoplus_{\delta \in(E \cap \mu)} \mathbf{Q} y_{\delta}\right) .
$$

Now suppose $A=A_{0} \oplus A_{1}$, where $\Gamma\left(A_{i}\right)=\tilde{E}_{i}(i=0,1)$. Define $A_{i, \nu}=A_{i} \cap A_{\nu}$ $\left(i=0,1 ; \nu<\omega_{1}\right)$. For $i=0,1$, since $\Gamma\left(A_{i}\right)=\tilde{E}_{i}$, there is a cub $\bigodot_{i}$ such that for $\nu \in \mathcal{C}_{i}, \nu \in E_{i}$ iff $A_{i} / A_{i, \nu}$ is not $\omega_{1}$-free. Let $\mathcal{C}=\bigodot_{0} \cap \bigodot_{1} \cap\left\{\nu<\omega_{1}: A_{\nu}=A_{0, \nu} \oplus\right.$ $\left.A_{1, \nu}\right\}$. Then $\circlearrowright$ is a cub, so by (*) there exists $\delta \in \circlearrowright \cap E_{0}$ such that for arbitrarily large $n, \eta_{\delta}(n) \in \bigodot \cap E_{1}$. Now

$$
A / A_{\delta}=A_{0} / A_{0, \delta} \oplus A_{1} / A_{1, \delta}
$$

where $A_{0} / A_{0, \delta}$ is not $\omega_{1}$-free and $A_{1} / A_{1, \delta}$ is $\omega_{1}$-free. Hence, since $y_{\delta}$ is divisible by all primes $\bmod A_{\delta}, y_{\delta}=a_{0}+a_{1}$ where $a_{0} \in A_{0}$ and $a_{1} \in A_{1, \delta}$. Choose $n$ so that $\eta_{\delta}(n)$ ( $=\gamma$, say) $\in E_{1}$ and $a_{1} \in A_{1, \gamma}$. Now $z_{\gamma, n}$ is divisible by infinitely many primes $\bmod A_{\gamma}$, so, just as above, $z_{\gamma, n}=u_{0}+u_{1}$ where $u_{1} \in A_{1}$ and $u_{0} \in A_{0, \gamma}$. By construction, $p_{n}$ divides $y_{\delta}-z_{\gamma, n}$ in $A$, i.e., $p_{n}$ divides $\left(a_{0}-u_{0}\right)+\left(a_{1}-u_{1}\right)$ in $A_{0} \oplus A_{1}$. Therefore, $p_{n} \mid\left(a_{0}-u_{0}\right)$ in $A_{0}$; hence, $p_{n} \mid a_{0} \bmod A_{\gamma}$ and thus, since $a_{1} \in A_{\gamma}, p_{n}$ divides $a_{0}+a_{1}=y_{\delta} \bmod A_{\gamma}$. But then, since $p_{n}\left|y_{\delta}-z_{\gamma, n}, p_{n}\right| z_{\gamma, n} \bmod A_{\gamma}$, which is impossible by construction. This contradiction completes the proof of (1).

(2) We shall use the model in Theorem 0.8(2). Let $A=\cup_{\nu<\omega_{1}} A_{\nu}, E, \bar{y}_{\delta}$ and $\left\{\eta_{\delta}\right.$ : $\delta \in E\}$ be as in Definition 2.3. Then in the model of $0.8(2)$ there is a cub $C$ such that for all $\delta \in E$, for all sufficiently large $n, \eta_{\delta}(n) \notin \bigodot$. Given $\tilde{E}=\bigvee_{\beta<\omega_{1}} \tilde{E}^{\beta}$ as in 2.9, we can assume $E=\amalg_{\beta<\omega_{1}} E^{\beta}$ where $\delta \in E^{\beta}$ implies $\delta>\beta$. Let $B^{\beta}$ be as in 2.7 for $E^{\prime}=E^{\beta}$. If $E \subseteq \bigodot$ we can immediately apply Theorem 2.4 and obtain the desired result. In general, though, we must apply a slight variation of the argument. By similar methods to those used in the proof of 2.4 we can prove that $A$ is $\mathcal{C}$-filtrationequivalent to $B=\bigoplus_{\beta<\omega_{1}} B^{\beta}$, i.e., for every $\nu \leqslant \omega_{1}$, there is an isomorphism $f_{\nu}$ : $A_{\nu} \rightarrow B_{\nu}$ such that for all $\mu<\nu$, if $\mu \in \mathcal{C}, f\left(A_{\mu}\right)=B_{\mu}$. In fact, the proof is somewhat simplified, because in Case 3, if $\nu=\delta \in \bigodot \cap E$, we can assume that $m=-1$, i.e., there are no elements $x_{0}, \ldots, x_{m}$ in $A_{\delta+1}$ pure independent over $A_{\delta}$ which have to be taken to themselves by $\tilde{f}$ (because for all $\tau \in \mathcal{C}, \tau>\delta, \eta_{\tau}$ can be chosen so that for all $n, \eta_{\tau}(n) \notin \mathcal{C}$. There will be a Case 4: $\nu \in E-\mathcal{C}$, which will be easy since there is a largest $\tau<\nu$ such that we require $f\left(A_{\tau}\right)=B_{\tau}$ ). Inspection of the proof of Theorem 1.2 shows that, assuming $\mathrm{MA}+\neg \mathrm{CH}$, e-filtration-equivalence implies isomorphism.

By similar methods one can prove the following, which says that the "associated divisibility function" for a group of type $R$ may or may not determine the group up to isomorphism in models of MA $+{ }_{\neg} \mathrm{CH}$ (cf. Theorem 1.4 and Remark 1.5).

2.11. CoRollary. The following is undecidable in $\mathrm{ZFC}+\mathrm{MA}+{ }_{\neg} \mathrm{CH}$ :

For all $\omega_{1}$-separable groups $A$ and $A^{\prime}$ of type $R, A$ is isomorphic to $A^{\prime}$ provided there are a stationary set $E, \omega_{1}$-filtrations $A=\cup_{\nu<\omega_{1}} A_{\nu}$ and $A^{\prime}=\cup_{\nu<\omega_{1}} A_{\nu}^{\prime}$, and elements $y_{\delta} \in A_{\delta+1}, y_{\delta}^{\prime} \in A_{\delta+1}^{\prime}(\delta \in E)$ such that $\left\{\nu<\omega_{1}: A_{\nu}\right.$ is not $\omega_{1}$-pure in $\left.A\right\}=E=\{\nu<$ $\omega_{1}: A_{\nu}^{\prime}$ is not $\omega_{1}$-pure in $\left.A^{\prime}\right\} ;\left\langle y_{\delta}, A_{\delta}\right\rangle_{*} / A_{\delta} \cong\left\langle y_{\delta}^{\prime}, A_{\delta}^{\prime}\right\rangle_{*} / A_{\delta}^{\prime} \cong R$, and for all $n \in \omega$, all $\mu<\delta, p_{n} \mid y_{\delta} \bmod A_{\mu+1}$ iff $p_{n} \mid y_{\delta}^{\prime} \bmod A_{\mu+1}^{\prime}$. 
Added in revision. An alternate proof of Theorem 2.8 (which avoids the construction in 2.7) can be given as follows. If $A, \bar{y}_{\delta},\left\{\eta_{\delta}: \delta \in E\right\}$ and $E=\amalg_{\beta<\omega_{1}} E^{\beta}$ are as in 2.8, let $B^{\beta}$ be the pure closure of $\left\{\bar{y}_{\delta}: \delta \in E^{\beta}\right\}$ and $B_{\nu}^{\beta}=B^{\beta} \cap A_{\nu}$. Since $B^{\beta}$ is pure in $A, e^{\beta} \stackrel{\text { def }}{=}\left\{\nu \mid B^{\beta}+A_{\nu}\right.$ is pure in $\left.A\right\}$ is a cub; moreover, without loss of generality, if $\nu<\mu$ in $\bigodot^{\beta}, B_{\mu}^{\beta} / B_{\nu}^{\beta} \cong B_{\mu}^{\beta} / B_{\nu}^{\beta} \oplus \mathbf{Z}^{(\omega)}$. If $\bigodot=$ the diagonal intersection

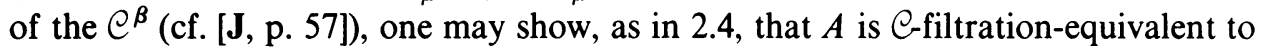
$\bigoplus_{\beta} B^{\beta}$.

For a simpler approach to the proof of 2.5 and 2.6 see the proof of Theorem 2.2 in the paper by Eklof and Mekler in the Proceedings of the Honolulu Conference on Abelian Groups, Springer-Verlag Lecture Notes in Mathematics.

3. Models of CH. In order to prove that the classification theorem (Theorem 1.2) fails if $2^{\aleph_{0}}<2^{\aleph_{1}}$ we shall make use of results of Devlin and Shelah on weak diamond. We say that a stationary set $E \subseteq \operatorname{Lim}\left(\omega_{1}\right)$ is nonsmall if the following is true.

$\Phi(E)$ : given for each $\nu \in E$ a partition $F_{\nu}: \mathcal{P}(\nu) \rightarrow\{0,1\}$ of the subsets of $\nu$ into 2 classes, there is a function $\varphi: E \rightarrow\{0,1\}$-called a weak diamond function for $\left\{F_{\nu}: \nu \in E\right\}$-such that for all $X \subseteq \omega_{1}$, $\left\{\nu \in E: F_{\nu}(X \cap \nu)=\varphi(\nu)\right\}$ is stationary.

Devlin and Shelah [DS] proved that $2^{\aleph_{0}}<2^{\aleph_{1}}$ implies that $\operatorname{Lim}\left(\omega_{1}\right)$ is nonsmall.

We shall prove that the classification theorem fails for groups in $\Gamma^{-1}(\tilde{E})$ when $\tilde{E}$ is nonsmall. All of our counterexamples will be of the kind described in the following lemma.

3.1. LemMA. Let $E \subseteq \operatorname{Lim}\left(\omega_{1}\right)$ be stationary and let $\left\{\eta_{\delta}: \delta \in E\right\}$ be a ladder system on $E$ such that for all $\delta \in E, n \in \omega, \eta_{\delta}(n)$ is a successor ordinal. Define

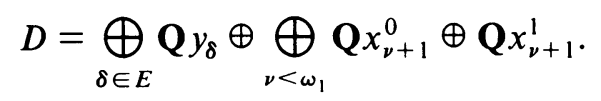

For each $\delta \in E, n \in \omega$ let $a_{n, \delta} \in\left\{x_{\eta_{\delta}(n)}^{0}, x_{\eta_{\delta}(n)}^{1}\right\}$. Fix a prime $p$ and for each $\delta \in E$, $n \in \omega$ let

$$
z_{n, \delta}=\frac{y_{\delta}-\sum_{i=0}^{n} p^{i} a_{i, \delta}}{p^{n+1}} .
$$

For each $\mu \leqslant \omega_{1}$ let $A_{\mu}$ be the subgroup of $D$ generated by $\left\{x_{\nu}^{l}: \nu<\mu, l=0\right.$, 1) $\cup\left\{z_{n, \delta}: \delta \in E \cap \mu, n \in \omega\right\}$. Then $A \stackrel{\text { def }}{=} A_{\omega_{1}}$ is an $\omega_{1}$-separable group of type $Q^{(p)}$ such that $\Gamma(A)=\tilde{E}$; moreover, for all $\delta \in E$ and all $n \in \omega, p^{n+1} \mid y_{\delta} \bmod A_{\mu+1}$ iff $\mu \geqslant \eta_{\delta}(n)$.

Proof. We claim that for all $\mu<\omega_{1}, A_{\mu+1}$ is a direct summand of $A$. For all $\delta \in E$ such that $\delta>\mu$ let $N_{\delta}$ be maximal such that $\eta_{\delta}\left(N_{\delta}\right) \leqslant \mu$; then $A=A_{\mu+1} \oplus C$, where

$$
C=\left\langle\left\{x_{\nu+1}^{l}: \nu \geqslant \mu, l=0,1\right\} \cup\left\{z_{\delta, n}: \delta \in E, \delta>\mu, n \geqslant N_{\delta}\right\}\right\rangle
$$


Then it is easy to see that $A$ is of type $Q^{(p)}$ with $\Gamma(A)=\tilde{E}$; in particular, if $\delta \in E$, $A_{\delta+1} / A_{\delta} \cong\left\langle\left\{y_{\delta}\right\} \cup A_{\delta}\right\rangle_{*} / A_{\delta} \cong Q^{(p)}$. By comparing coefficients in $D$ (cf. [E, proofs of $8.2,11.1]$ ) one can prove the $x_{\nu}^{l}$ for $v \geqslant \mu$ are pure-independent $\bmod A_{\mu}$, as well as the final assertion of the lemma.

3.2. THEOREM. If $E \subseteq \operatorname{Lim}\left(\omega_{1}\right)$ is nonsmall, there exist $\omega_{1}$-separable groups $A$ and $B$ with $\Gamma(A)=\Gamma(B)=\tilde{E}$ such that $A$ and $B$ are filtration-equivalent but not isomorphic.

Proof. Fix a ladder system $\left\{\eta_{\delta}: \delta \in E\right\}$ on $E$ s.t. every $\eta_{\delta}(n)$ is a successor; $A$ and $B$ will be defined as in 3.1 using this ladder system. By the last part of Lemma 3.1 and by Theorem 1.4, $A$ and $B$ will be filtration-equivalent. (Note that the associated divisibility system $\mathscr{D}$-see before Theorem 1.4 -is given by $\mathscr{D}(\delta)=\left(\eta_{\delta}, k_{\delta}\right)$ where $k_{\delta}(n)=n+1$ for all $n \in \omega$.) Define $b_{n, \delta}=x_{\eta_{\delta}(n)}^{0}$ for all $n \in \omega, \delta \in E$ and let $B$ be defined as in Lemma 3.1 using these elements. We shall use $\Phi(E)$ in order to choose elements $a_{n, \delta}$ so that if $A$ is defined using these elements, then $A \not B$.

For all $\sigma$, let $D_{\sigma}$ be the $\mathbf{Q}$-submodule of $D$ generated by $\left\{x_{\nu+1}^{l}: \nu<\sigma, l=0,1\right\} \cup$ $\left\{y_{\tau}: \tau<\sigma\right\}$. Suppose that $Y$ is a Z Z-submodule of $D_{\delta}$ containing $\left\{x_{\nu+1}^{l}: \nu<\delta\right.$, $l=0,1\}$ s.t. $\forall \mu<\delta,\left\{x_{\nu}^{l}: \nu \geqslant \mu\right\}$ is pure-independent $\bmod Y_{\mu} \stackrel{\text { def }}{=} Y \cap D_{\mu}$. For $l=0,1$, let $a_{n, \delta}^{l}=x_{\eta_{\delta}(n)}^{l}$ and let $Y^{l}=\left\langle Y \cup\left\{z_{n, \delta}^{l}: n \in \omega\right\}\right\rangle$, where $z_{n, \delta}^{l}$ are defined as in (3.1.1) using the $a_{n, \delta}^{l}$. We claim that

there is no isomorphism of $B_{\delta}$ onto $Y$ which extends both to a monomorphism of $B_{\delta+1}$ into $Y^{0}$ and to a monomorphism of $B_{\delta+1}$ into $Y^{1}$.

Supposing the claim to be true we shall describe the construction of $A$. If $Y$ is a subset of $D_{\delta}$ and $\theta \subseteq B_{\delta} \times D_{\delta}$, define $F_{\delta}(Y, \theta)=0$ if $Y$ is a Z-submodule of $D_{\delta}$ as above and $\theta$ is an isomorphism of $B_{\delta}$ onto $Y$ which extends to a monomorphism: $B_{\delta+1} \rightarrow Y^{1}$; define $F_{\delta}(Y, \theta)=1$ in all other cases. By $\Phi(E)$ there is a function $\varphi$ : $E \rightarrow\{0,1\}$ such that for all $A \subseteq D$ and $\Theta \subseteq B \times D$,

$$
\left\{\delta \in E: \varphi(\delta)=F_{\delta}\left(A \cap D_{\delta}, \Theta \cap\left(B_{\delta} \times D_{\delta}\right)\right)\right\}
$$

is stationary in $E$.

Now, using $\varphi$, we will inductively define $A_{\nu} \subseteq D_{\nu}$ so that $A \stackrel{\text { def }}{=} \cup_{\nu<\omega_{1}} A_{\nu}$ is a subgroup of $D$ as defined in 3.1. Suppose $A_{\nu}$ has been defined for all $\nu<\mu$. If $\mu$ is a limit ordinal, let $A_{\mu}=\cup_{\nu<\mu} A_{\nu}$. If $\mu=\nu+1$, where $\nu \notin E$, let $A_{\mu}=\left\langle A_{\nu} \cup\right.$ $\left.\left\{x_{\nu}^{0}, x_{\nu}^{1}\right\}\right\rangle$ (or $A_{\mu}=A_{\nu}$ if $\nu \in \operatorname{Lim}\left(\omega_{1}\right)-E$ ). If $\mu=\delta+1$, where $\delta \in E$, let $Y=A_{\delta}$ and let $A_{\mu}=Y^{\varphi(\delta)}$. We want to prove that $B$ is not isomorphic to $A$. Suppose, to the contrary, that there is an isomorphism $\Theta: B \rightarrow A$. Then the set of $\nu$ such that $\Theta\left(B_{\nu}\right)=A_{\nu}$ is a cub so there exists $\delta \in E$ such that $\Theta\left(B_{\delta}\right)=A_{\delta}$ and $\varphi(\delta)=$ $F_{\delta}\left(A_{\delta}, \Theta \cap\left(B_{\delta} \times D_{\delta}\right)\right)$. Notice that $\Theta \cap\left(B_{\delta} \times D_{\delta}\right)=\Theta \mid B_{\delta}$, which is an isomorphism: $B_{\delta} \rightarrow A_{\delta}$; call it $\theta$. Let $Y=A_{\delta}$. First suppose $\varphi(\delta)=0$, so $A_{\delta+1}=Y^{0}$. Then by definition of $F_{\delta}, \theta$ extends to a monomorphism: $B_{\delta+1} \rightarrow Y^{1}$. But $\theta\left(y_{\delta}\right) \in A_{\delta+1}$ (since $\theta\left(y_{\delta}\right)$ is $p$-divisible $\bmod A_{\delta}$ ) so $\theta$ extends to a monomorphism: $B_{\delta+1} \rightarrow Y^{0}=$ $A_{\delta+1}$, viz $\Theta \uparrow B_{\delta+1}$. This contradicts claim (3.2.1). We also obtain a contradiction if $\varphi(\delta)=1$, since then $\Theta \uparrow B_{\delta+1}: B_{\delta+1} \rightarrow Y^{1}=A_{\delta+1}$ demonstrates that $F_{\delta}(Y, \theta)=0$. 
Thus it remains only to prove claim (3.2.1). Suppose that $f: B_{\delta} \rightarrow Y$ is an isomorphism and $f_{l}: B_{\delta+1} \rightarrow Y^{l}(l=0,1)$ are monomorphisms extending $f$. Then for some $m$ there exist $k_{0}, k_{1} \in \omega$ and $u_{0}, u_{1} \in A_{\delta}$ such that $f_{l}\left(p^{m} y_{\delta}\right)=p^{k_{l}} y_{\delta}+u_{l} \in Y^{l}$. Say $k_{1} \geqslant k_{0}$; choose $r \in \omega$ such that $u_{0}, u_{1} \in Y_{\eta_{\delta}(r)}$, and let $n=r+k_{0}+1$. Then $p^{n}$ divides $y_{\delta}-\sum_{i=0}^{n-1} p^{i} b_{i, \delta}$ in $B_{\delta+1}$, so $p^{n+m}$ divides

$$
p^{k_{\prime}} y_{\delta}+u_{l}-p^{m} \sum_{i=0}^{n-1} p^{i} f\left(b_{i, \delta}\right)
$$

in $Y^{l}$. But also $p^{n}$ divides

$$
y_{\delta}-\sum_{i=0}^{n-1} p^{i} x_{\eta_{\delta}(i)}^{\prime}
$$

in $Y^{\prime}$, so (subtracting) $p^{n}$ divides

$$
u_{l}-p^{r} \sum_{i=0}^{n-1} p^{i} f\left(b_{i, \delta}\right)+p^{k} \sum_{i=0}^{n-1} p^{i} x_{\eta_{\delta}(i)}^{l}
$$

in $Y^{l}$ and hence in $Y$. Therefore, $p^{n}$ divides $\left(u_{1}-u_{0}\right)-p^{k} \sum_{i=0}^{n-1} p^{i}\left(p^{d} x_{\eta_{\delta}(i)}^{1}-x_{\eta_{\delta}(i)}^{0}\right)$ in $Y$, where $d=k_{1}-k_{0}$. But this is impossible as the coefficient of $x_{\eta_{\delta}(r)}^{0}$ is $p^{k_{0}+r}<p^{n}$ and the $\left\{x_{\nu}^{l}: \nu \geqslant \eta_{\delta}(r)\right\}$ are pure-independent $\bmod Y_{\eta_{\delta}(r)}$.

3.3. RemarKs. (1) By a slight modification we can even get that for all free $F$, $A \oplus F \not B \oplus F$. It may be argued that Theorem 3.2 is strong evidence for the claim that in a model of $\mathrm{CH}$ there is no possible meaningful classification of all $\omega_{1}$-separable groups. It is difficult to see what conceivable scheme of classification could distinguish between filtration-equivalent groups-in particular, between the groups $A$ and $B$ constructed in the proof of 3.2 .

(2) Theorem 3.2 may be strengthened: if $E$ is nonsmall, there is a family of $2^{\aleph_{1}}$ $\omega_{1}$-separable groups $A_{i}\left(i<2^{\aleph_{1}}\right)$ in $\Gamma^{-1}(\tilde{E})$ such that, for all $i \neq j, A_{i}$ and $A_{j}$ are filtration-equivalent but not isomorphic. The proof uses the fact that every nonsmall $E$ is the disjoint union of $\boldsymbol{\aleph}_{1}$ nonsmall subsets (see e.g., [EH, Lemma 2.8]).

(3) In a model of $\mathrm{CH}$ there are (by the last sentence of (2)) many-in fact $2^{\aleph_{1}}$-classes $\tilde{E}$ such that the classification theorem fails for groups in $\Gamma^{-1}(\tilde{E})$. In some models of $\mathrm{CH}$ (e.g. $L$ ) every stationary set is nonsmall, so the classification theorem fails completely. However, Shelah [S1] has shown that there are models of $\mathrm{GCH}$ in which some stationary sets are small. Using his methods we shall prove the following result which shows that in some models of $\mathrm{GCH}$ the classification theorem may be partially salvaged.

3.4. THEOREM. There is a model of $Z F C+G C H$ such that there exists a stationary and costationary set $S \subseteq \omega_{1}$ such that (i) whenever $A$ and $B$ are filtration-equivalent and $\Gamma(A) \subseteq \tilde{S}, A$ and $B$ are isomorphic; but (ii) for every $E \subseteq \omega_{1}$ with $\tilde{E} \nsubseteq \tilde{S}$, there are $\omega_{1}$-separable groups $A$ and $B$ with $\Gamma(A)=\tilde{E}=\Gamma(B)$ which are filtration-equivalent but not isomorphic.

Proof. We use the model described in Theorem 0.7(4). If $A$ and $B$ are as in (i), let $\mathbf{P}$ be the set of all level-preserving $f: A_{\nu+1} \rightarrow B_{\nu+1}$ partially-ordered by $f^{\prime} \leqslant f$ iff 
$f^{\prime} \supseteq f$. Then one may prove that $\mathbf{P}$ is proper and $\left(\omega_{1}-S\right)$-complete (cf. [M2]), so if $D_{\nu}=\left\{f \in \mathbf{P}\right.$ : Dom $\left.f \supseteq A_{\nu+1}\right\}$, there is a pairwise compatible subset $G$ which interests each $D_{\nu}$. Then $\cup G$ is an isomorphism: $A \rightarrow B$.

Part (ii) follows from the proof of Theorem 3.2, because if $\tilde{E} \nsubseteq \tilde{S}, \diamond(E)$ holds (so a fortiori $\Phi(E)$ holds).

Next we shall show, using diamond, that the direct decomposition theorems of $\S 2$ fail. (We do not know if any of these theorems are consistent with $\mathrm{CH}$.)

3.5. TheOREM. ${ }^{2}$ Assume $\diamond(E)$ for some stationary subset $E \subseteq \operatorname{Lim}\left(\omega_{1}\right)$. Then there is an $\omega_{1}$-separable group $A=\cup_{\nu<\omega_{1}} A_{\nu}$ of cardinality $\omega_{1}$ such that $\Gamma(A)=\tilde{E}$ and $A$ is not the direct sum of two uncountable groups.

Proof. We shall define by induction on $\nu<\omega_{1}$ a chain of groups $A_{\nu}$ and subgroups $B_{\tau \nu}$ for $\tau \in \nu-E$ such that $A_{\tau} \oplus B_{\tau \nu}=A_{\nu}$ and for all $\tau<\mu<\nu(\tau, \mu \in \nu$ $-E), B_{\tau \mu} \oplus B_{\mu \nu}=B_{\tau \nu}$. Moreover, we require that for all $\mu<\nu$,

$$
A_{\nu} / A_{\mu} \cong \begin{cases}Q^{(p)} & \text { if } \mu \in E, \\ \text { free } & \text { otherwise. }\end{cases}
$$

If we can do this then $\Gamma(A)=\tilde{E}$ and $A$ is $\omega_{1}$-separable since for all $\tau \notin E$, $A=A_{\tau} \oplus\left(\cup_{\tau<\nu<\omega_{1}} B_{\tau \nu}\right)$ (cf. [M1, pp. $\left.\left.1213 \mathrm{ff}\right]\right)$.

We shall use $\diamond(E)$ to do the construction so that $A$ is not the direct sum of 2 uncountable subgroups. (The methods are an extension of those in [EM or $\mathbf{E}$, Chapter 9]. I am grateful to A. Mekler for help with the case of $\tilde{E}=1$.) We will always construct $A_{\nu}$ to be a subgroup of $D_{\nu}$ (see proof of 3.2). By $\diamond(E)$ there exist subsets $Y_{\delta}$ and $Y_{\delta}^{\prime}$ of $D_{\delta}$ (for $\delta \in E$ ) such that for all $Z, Z^{\prime} \subseteq D,\left\{\delta \in E: Z \cap D_{\delta}=Y_{\delta}\right.$ and $\left.Z^{\prime} \cap D_{\delta}=Y_{\delta}^{\prime}\right\}$ is stationary.

The only nontrivial case in the inductive construction is the following: $A_{\delta}$ has been defined and (*) $\delta \in E$ and $Y_{\delta}$ and $Y_{\delta}^{\prime}$ are subgroups of $A_{\delta}$, and there is a ladder $\eta_{\delta}$ on $\delta$ such that for all $n \in \omega$, letting $\tau_{n}=\eta_{\delta}(n)$ :

$$
\begin{aligned}
& A_{\tau_{n}}=\left(Y_{\delta} \cap A_{\tau_{n}}\right) \oplus\left(Y_{\delta}^{\prime} \cap A_{\tau_{n}}\right) ; \\
& Y_{\delta} \cap A_{\tau_{n+1}} / Y_{\delta} \cap A_{\tau_{n}} \text { has a summand } \cong \mathbf{Z} ; \text { and } \\
& Y_{\delta}^{\prime} \cap A_{\tau_{n+1}} / Y_{\delta}^{\prime} \cap A_{\tau_{n}} \text { has a summand } \cong \mathbf{Z} .
\end{aligned}
$$

For each $n \in \omega$ we shall define $c_{n} \in Y_{\delta} \cap A_{\tau_{2 n}}$ and $c_{n}^{\prime} \in Y_{\delta}^{\prime} \cap A_{\tau_{2 n}}$ such that $c_{n}+c_{n}^{\prime} \in B_{\tau_{2 n-2}+1, \tau_{2 n}}$ and $c_{n}$ (resp. $c_{n}^{\prime}$ ) is of height $1 \bmod Y_{\delta} \cap A_{\tau_{2 n-1}}$ (resp. $Y_{\delta}^{\prime} \cap$ $A_{\tau_{, n-1}}$ ). Then letting $b_{n}=c_{n}+c_{n}^{\prime}$, define

$$
z_{n}=\frac{y_{\delta}-\sum_{i=0}^{n} p^{i} b_{i}}{p^{n+1}}
$$

and $A_{\delta+1}=\left\langle A_{\delta} \cup\left\{z_{n}: n \in \omega\right\}\right\rangle$. Thus, if for each $n$ we let

$$
B^{n}=\left\langle B_{\tau_{2 n}+1, \delta} \cup\left\{z_{m}: m \geqslant n\right\}\right\rangle
$$

\footnotetext{
${ }^{2}$ This result has been improved by Mekler, who, assuming $\diamond(E)$, has constructed an $\omega_{1}$-separable group $A$ which is "almost endo-rigid" i.e., any endomorphism is, modulo a countable summand, multiplication by an integer. See also Theorem 1.3 in the paper by Eklof and Mekler in the Proceedings of the Honolulu Conference on Abelian Groups, Springer-Verlag Lecture Notes in Mathematics.
} 
we have

$$
A_{\delta+1}=A_{\tau_{2 n}+1} \oplus B^{n}
$$

and so, if for $\mu \in\left(\tau_{2 n}+1\right)-E$, we let $B_{\mu, \delta+1}=B_{\mu . \tau_{2 n}+1} \oplus B^{n}$ we have

$$
A_{\delta+1}=A_{\mu} \oplus B_{\mu, \delta+1} \text {. }
$$

Now by hypothesis there exists $\tilde{c}_{n} \in Y_{\delta} \cap A_{\tau_{2 n}}$ (resp. $\tilde{c}_{n}^{\prime} \in Y_{\delta}^{\prime} \cap A_{\tau_{2 n}}$ ) which is of height $1 \bmod Y_{\delta} \cap A_{\tau_{2 n-1}}\left(\right.$ resp. $Y_{\delta}^{\prime} \cap A_{\tau_{2 n-1}}$ ). Since $A_{\tau_{2 n}}=A_{\tau_{2 n-2}+1} \oplus B_{\tau_{2 n-2}+1 . \tau_{2 n}}$, there exists $a \in A_{\tau_{2 n-2}+1} \subseteq A_{\tau_{2 n-1}}$ s.t. $\tilde{c}_{n}+\tilde{c}_{n}^{\prime}+a \in B_{\tau_{2 n-2}+1 . \tau_{2 n}}$. But since $A_{\tau_{2 n-1}}=$ $\left(Y_{\delta} \cap A_{\tau_{2 n-1}}\right) \oplus\left(Y_{\delta}^{\prime} \cap A_{\tau_{2 n-1}}\right)$ there exists $u \in Y_{\delta} \cap A_{\tau_{2 n-1}}\left(u^{\prime} \in Y_{\delta}^{\prime} \cap A_{\tau_{2 n-1}}\right)$ such that $a=u+u^{\prime}$. Then let $c_{n}=\tilde{c}_{n}+u, c_{n}^{\prime}=\tilde{c}_{n}^{\prime}+u^{\prime}$.

This completes the construction. We claim that $A$ is not the direct sum of two uncountable groups. Suppose to the contrary, that $A=H \oplus H^{\prime}$ where $H$ and $H^{\prime}$ are uncountable. Then

$$
\circlearrowright \stackrel{\text { def }}{=}\left\{\nu<\omega_{1}: A_{\nu}=\left(H \cap A_{\nu}\right) \oplus\left(H^{\prime} \cap A_{\nu}\right)\right\}
$$

is a cub. Also

$$
S \stackrel{\text { def }}{=}\left\{\nu<\omega_{1}: H \cap A_{\nu+1} / H \cap A_{\nu} \text { has a summand } \cong \mathbf{Z}\right\}
$$

and

$$
S^{\prime} \stackrel{\text { def }}{=}\left\{\nu<\omega_{1}: H^{\prime} \cap A_{\nu+1} / H^{\prime} \cap A_{\nu} \text { has a summand } \cong \mathbf{Z}\right\}
$$

are unbounded. Thus their closures, $\bar{S}$ and $\overline{S^{\prime}}$, are cubs. Hence, there exists $\delta \in E$ which is the limit of points

$$
\tau_{n} \in \mathcal{S} \cap \cap S^{\prime}
$$

and satisfies $H \cap A_{\delta}=Y_{\delta}, H^{\prime} \cap A_{\delta}=Y_{\delta}^{\prime}$ (cf. [EM]). Thus, we are in the crucial case when (*) holds. Now $y_{\delta}=h+h^{\prime}$ where $h \in H \cap A_{\mu}, h^{\prime} \in H \cap A_{\mu}^{\prime}$ for some $\mu \in \mathcal{C}$, $\mu \geqslant \delta+1$. Since

$$
A_{\mu} / A_{\delta}=\left(H \cap A_{\mu} / H \cap A_{\delta}\right) \oplus\left(H^{\prime} \cap A_{\mu} / H^{\prime} \cap A_{\delta}\right) \cong Q^{(p)} \oplus \mathbf{Z}^{(\omega)}
$$

and $y_{\delta}$ is $p$-divisible $\bmod A_{\delta}$, either $h \in A_{\delta}$ or $h^{\prime} \in A_{\delta}$. Say $h \in A_{\delta}$. Pick $n$ large enough so that $h \in A_{\tau_{2 n-1}}$. Now $p^{n+1}$ divides

$$
\left(h-\sum_{i=0}^{n} p^{i} c_{i}\right)+\left(h^{\prime}-\sum_{i=0}^{n} p^{i} c_{i}^{\prime}\right) \in H \oplus H^{\prime} .
$$

Thus $p^{n+1}$ divides

$$
\left(h-\sum_{i=0}^{n} p^{i} c_{i}\right)
$$


in $H$. But then $p^{n+1}$ divides $\sum_{i=0}^{n} p^{i} c_{i} \bmod A_{\tau_{2 n-1}}$; so $p^{n+1} \operatorname{divides} p^{n} c_{n} \bmod A_{\tau_{2 n-1}}$ (since $\sum_{i=0}^{n-1} p^{i} c_{i} \in A_{\tau_{2 n-2}}$ ). This contradicts the fact that $c_{n}$ has height $1 \bmod A_{\tau_{2 n-1}}$.

An immediate consequence is that 2.6 fails.

3.6. COROLlaRy $(V=L)$. For every stationary $E \subseteq \omega_{1}$ there exists an $\omega_{1}$-separable group $A \subset \Gamma^{-1}(\tilde{E})$ such that $A$ is not isomorphic to $A \oplus \mathbf{Z}^{\left(\omega_{1}\right)}$.

Proof. If $A$ is as in Theorem 3.5, $A \not A \oplus \mathbf{Z}^{\left(\omega_{1}\right)}$.

We can also show that 2.5 fails, even assuming $\mathrm{CH}$ :

3.7. ThEOREM $(\Phi(E))$. Every $\omega_{1}$-separable group $A$ of cardinality $\omega_{1}$ such that $\Gamma(A) \supseteq \tilde{E}$ contains a subgroup $B$ such that $A / B$ is countable, but $B$ is not $\omega_{1}$-separable.

Proof. Since $\Gamma(A) \supseteq \tilde{E}, \Phi(E)$ implies $\operatorname{Ext}(A, \mathbf{Z}) \neq 0$ (cf. [E, Theorem 3.6]). Thus $A$ is not $\omega_{1}$-coseparable, so by [G2, Theorem 193] $A$ is not totally $\omega_{1}$-separable; in fact, an inspection of the proof shows that there is a $B$ such that $A / B$ is countable but $B$ is not $\omega_{1}$-separable.

As in Theorem 3.4 we can obtain models of $\mathrm{GCH}$ where the decomposition property holds in some classes $\Gamma^{-1}(\tilde{E})$ and fails in others.

3.8. THEOREM. There is a model of $Z F C+G C H$ such that there exists a stationary and costationary set $S \subseteq \omega_{1}$ such that (i) every $\omega_{1}$-separable group $A$ such that $\Gamma(A) \subseteq \tilde{S}$ has the decomposition property; but (ii) for every $E \subseteq \omega_{1}$ such that $\tilde{E} \nsubseteq \tilde{S}$ there is an $\omega_{1}$-separable group $A$ with $\Gamma(A)=\tilde{E}$ which is not the direct sum of two uncountable groups.

Proof. We use the model described in Theorem 0.7(4). We can show that in this model, Theorem $0.8(2)(* *)$ holds for every stationary $E \subseteq S$; this is because the $\mathbf{P}$ defined in the proof of $0.8(2)$ is $\left(\omega_{1}-S\right)$-complete. [Note also that $\mathrm{CH}$ implies $|\mathbf{P}|=\aleph_{1}$.] Then using Theorem 3.4, (i) follows just as in Theorem 2.10(2). Furthermore, (ii) holds by Theorem 3.5, because in this model $\diamond(E)$ holds for all $E$ such that $\tilde{E} \nsubseteq \tilde{S}$.

\section{REFERENCES}

[B1] J. Baumgartner, Iterated forcing, Proc. 1978 Cambridge Set Theory Conf. (to appear).

[B2] __ Proper forcing, Handbook of Set Theoretic Topology (to appear).

[D] K. Devlin, The Yorkshireman's guide to proper forcing, Proc. 1978 Cambridge Set Theory Conf. (to appear).

[DS] K. Devlin and S. Shelah, $A$ weak version of $\diamond$ which follows from $2^{\aleph_{0}}<2^{\aleph_{1}}$, Israel J. Math. 29 (1978), 239-247.

[E] P. Eklof, Set theoretic methods in homological algebra and abelian groups, Presses Univ. Montréal, Montréal, 1980.

[EH] P. Eklof and M. Huber, On the rank of Ext, Math. Z. 174 (1980), 159-185.

[EM] P. Eklof and A. Mekler, On constructing indecomposable groups in L, J. Algebra 49 (1977), 96-103.

[EMS] P. Eklof, A. Mekler and S. Shelah, Almost disjoint groups, Israel J. Math. (to appear).

[F] L. Fuchs, Infinite abelian groups, vol. II, Academic Press, New York, 1973.

[G1] P. Griffith, A note on a theorem of Hill, Pacific J. Math. 29 (1969), 276-284.

[G2] __ Infinite abelian groups, Univ. of Chicago Press, Chicago, Ill., 1970.

[H] W. Hodges, Seminar on proper forcing, (lecture notes).

[J] T. Jech, Set theory, Academic Press, New York, 1978. 
[M1] A. Mekler, How to construct almost free groups, Canad. J. Math. 32 (1980), 1206-1228.

[M2] __ Shelah's Whitehead groups and CH, Rocky Mountain J. Math. 12 (1982), 271-278.

[S1] S. Shelah, Whitehead groups may be not free, even assuming CH. I, Israel J. Math. 28 (1977), 193-204.

[S2] , Proper forcing, Lecture Notes in Math., vol. 940, Springer-Verlag, Berlin and New York, 1982.

[S3] _. On endo-rigid, strongly $\aleph_{1}$-free abelian groups in $\aleph_{1}$, Israel J. Math. 40 (1981), 291-295.

[ST] R. Solovay and S. Tenenbaum, Iterated Cohen extensions and Souslin's problem, Ann. of Math. (2) 94 (1971), 201-245.

Department of Mathematics, University of California, Irvine, California 92717 